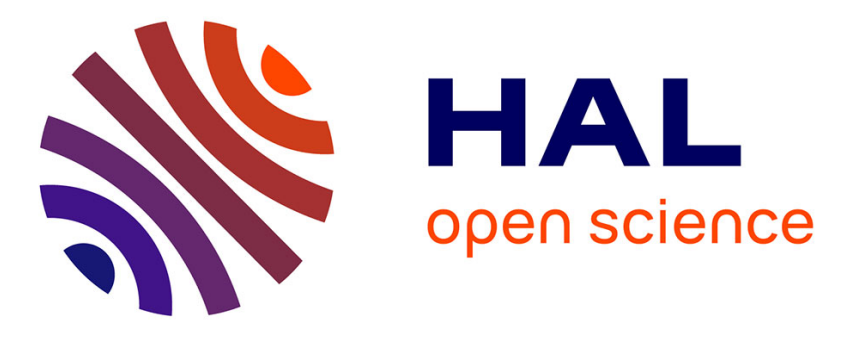

\title{
Magnetism of iron: from the bulk to the monoatomic wire
}

\author{
Gabriel Autes, Cyrille Barreteau, Daniel Spanjaard, Marie-Catherine \\ Desjonqueres
}

\section{To cite this version:}

Gabriel Autes, Cyrille Barreteau, Daniel Spanjaard, Marie-Catherine Desjonqueres. Magnetism of iron: from the bulk to the monoatomic wire. Journal of Physics: Condensed Matter, 2006, 18, pp.6785. 10.1088/0953-8984/18/29/018 . hal-00020095

\section{HAL Id: hal-00020095 \\ https://hal.science/hal-00020095}

Submitted on 6 Mar 2006

HAL is a multi-disciplinary open access archive for the deposit and dissemination of scientific research documents, whether they are published or not. The documents may come from teaching and research institutions in France or abroad, or from public or private research centers.
L'archive ouverte pluridisciplinaire $\mathbf{H A L}$, est destinée au dépôt et à la diffusion de documents scientifiques de niveau recherche, publiés ou non, émanant des établissements d'enseignement et de recherche français ou étrangers, des laboratoires publics ou privés. 


\title{
Magnetism of iron: from the bulk to the monoatomic wire
}

\author{
Gabriel Autès*, Cyrille Barreteau*, Daniel Spanjaard ${ }^{\dagger}$ and Marie-Catherine Desjonquères* \\ ${ }^{*}$ CEA Saclay, DSM/DRECAM/SPCSI, Bâtiment 462, F-91191 Gif sur Yvette, France and \\ ${ }^{\dagger}$ Laboratoire de Physique des Solides, Université Paris Sud, Bâtiment 510, F-91405 Orsay, France
}

(Dated: March 6, 2006)

The magnetic properties of iron (spin and orbital magnetic moments, magnetocrystalline anisotropy energy) in various geometries and dimensionalities are investigated by using a parametrized tight-binding model in an $s, p$ and $d$ atomic orbital basis set including spin polarization and the effect of spin-orbit coupling. The validity of this model is well established by comparing the results with those obtained by using an ab-initio code. This model is applied to the study of iron in bulk bcc and fcc phases, (110) and (001) surfaces and to the monatomic wire, at several interatomic distances. New results are derived. The variation of the component of the orbital magnetic moment on the spin quantization axis has been studied as a function of depth, revealing a significant enhancement in the first two layers, especially for the (001) surface. It is found that the magnetic anisotropy energy is drastically increased in the wire and can reach several meV. This is also true for the orbital moment, which in addition is highly anisotropic. Furthermore it is shown that when the spin quantization axis is neither parallel nor perpendicular to the wire the average orbital moment is not aligned with the spin quantization axis. At equilibrium distance the easy magnetization axis is along the wire but switches to the perpendicular direction under compression. The success of this model opens up the possibility of obtaining accurate results on other elements and systems with much more complex geometries.

PACS numbers: 71.15.Ap,71.20.Be,71.70.Ej,73.20.At,73.22.Dj,75.10.Lp,75.70.Rf,75.50.Bb,75.75.+a

\section{INTRODUCTION}

The magnetic properties of nanoparticles, thin films and wires have attracted recently a lot of attention due to their potential technological applications. It is thus very important to investigate the influence of dimensionality on these properties. In many experimental systems, some atoms are in a bulk-like environment while some others have a very low coordinence in a strongly asymmetric environment. This is the case of clusters, at the edge of adsorbed islands, supported wires, along step edges or in the nanoconstriction region of a break junction etc.. As a consequence the magnetic properties of such systems need to be treated at an atomic level, by studying their electronic structure in the framework of quantum mechanics.

In principle these properties can be determined from ab-initio calculations. However the computer time and storage increase drastically with the number of inequivalent atoms. Thus there is a need for simplified methods still based on quantum mechanics which capture the essential physics. In this context tight-binding (TB) methods are ideally suited for these calculations. Indeed TB methods can be extended to magnetic systems by introducing Hubbard two-body terms treated in the Hartree-Fock approximation [1, 2], and can easily include spin-orbit effects[3]. Moreover they allow the calculation of local physical quantities (spin or orbital moment etc..) in a straightforward manner which gives a physically transparent understanding of the phenomena. 
The anisotropy of the magneto-crystalline energy (MAE), although small in transition metals, is of fundamental importance since it determines the easy magnetization axis. The MAE results from the coupling of the spin and orbital moments. In the bulk it is well known that the orbital moment is nearly quenched due to the high symmetry of the potential. When dimensionality or symmetry is reduced the orbital moment is less and less quenched and the MAE increases rapidly. The MAE is routinely measured by magnetic hysteresis or torque measurements 4 , 5 , 6], for instance. More recently the development of X-ray magnetic circular dichroism techniques has allowed the experimental determination of the orbital moment[7]. On the theoretical side the calculation of the MAE in bulk pure ferromagnetic transition metals is a challenge because of its minuteness (typically some $\mu \mathrm{eV}$ ). The first attempts to determine the MAE on surfaces have been performed on free standing mono-layers either using a perturbative treatment of the spin-orbit coupling in a tight-binding model [6, 8), or with self-consistent ab-initio technique 9, 10]. The calculations performed by Bruno [6. 8] on mono-layers have been extended to study slabs with several atomic layers 11, 12 in a pure $d$-band model. More recently the case of layered ordered alloys of the CuAu or $\mathrm{CsCl}$ types containing at least one ferromagnetic element [13, deposited ferromagnetic over-layers on non-magnetic substrates 14, and multilayers have been investigated 15, 16. Simultaneously the orbital magnetism driven by the spin-orbit interaction has been computed using the tight-binding model (perturbatively 6 , 8 or non perturbatively 17 . 18]) as well as ab-initio codes 19, 20.

The aim of this paper is to develop a TB model allowing the determination of the magnetic properties of transition metals in various geometrical configurations, going from highly coordinated and symmetric environments to low coordinated and anisotropic geometries. In a first step we have found useful to check the validity of the model by a detailed comparison with the results provided by ab-initio methods on simple systems. In addition, the use of both methods yields a better physical understanding of these properties, and, moreover, the simplicity of our model allows a more detailed analysis of each system. We use a non-orthogonal basis set of $s, p$ and $d$ valence orbitals. The parametrization of the non-magnetic and non-relativistic Hamiltonian was derived by Mehl and Papaconstantopoulos 21. The possibility of spin polarization is then introduced using a Stoner like model in which the splitting between the energy levels of up and down spin orbitals is governed by the Stoner parameter $I_{d d^{\prime}}$ and is proportional to the magnetic moment carried by $d$ electrons. Indeed it is well known that $s$ and $p$ electrons are very weakly polarized. The relativistic effects, limited to spin-orbit coupling between $d$ electrons, are taken into account by adding the intra atomic matrix elements of this coupling determined by a parameter $\xi$. The two parameters $I_{d d^{\prime}}$ and $\xi$ are fixed by comparison with ab-initio calculations. For the Stoner parameter the variation of the magnetization as a function of interatomic distance in the bulk phase can be used. The determination of the spin-orbit coupling parameter relies on the study of the degeneracy removal of energy bands at high symmetry points or directions of the Brillouin zone.

We have applied our model to the study of iron in various atomic arrangements going from the bulk, in the experimentally observed phases bcc and fcc, to simple surfaces and finally to the monoatomic wire, at several interatomic distances. It is found that with a unique value of the Stoner parameter we are able to reproduce the variation of the spin magnetic moment in a wide range of lattice spacings in the bcc bulk phase. It is well known that the magnetic properties of bulk fcc iron are rather complex. In spite of this complexity the calculations carried out in this work are in excellent agreement with ab-initio predictions. The spin-orbit coupling parameter is then determined, as explained above, and a single value of this parameter is able to reproduce the ab-initio band structure. Furthermore the contribution of the orbital moment to the magnetization in bcc Fe is very close to the experimental value. The case 
of surfaces represents a more stringent check since the dimensionality is reduced and all atoms are not geometrically equivalent. It is in particular interesting to follow the variation of the magnetic properties when going from the outermost to inner (bulk-like) layers. The spin-polarized surface projected band structure as well as the variation of the spin magnetic moment as a function of depth derived from ab-initio calculations are perfectly reproduced by our model. In particular the (001) surface atoms have a saturated moment contrary to those of the (110) surface. Introducing the spin-orbit coupling in the TB Hamiltonian allows the calculation of the MAE and of the orbital magnetic moment at each inequivalent site. Due to the efficiency of our model it is possible to check rigorously the convergence of these quantities when increasing the number of $k$ points. The orbital moment is strongly increased on surface atoms (about twice the bulk value for the (001) surface), and recovers its bulk value on the third and innermost layers. The wire is the most anisotropic atomic arrangement with the lowest coordinence. Even though the TB parameters are fitted on bulk ab-initio data only, the non-magnetic band structure calculated with these parameters is in good agreement with ab-initio calculations, proving their good transferability. The two additional parameters $I_{d d^{\prime}}$ and $\xi$ are also perfectly transferable. Indeed, without changing the Stoner parameter, the variation of the spin magnetic moment with the interatomic distance is satisfactorily reproduced (in particular the saturated solution appears abruptly at the same interatomic spacing) and the splitting of bands due to spin-orbit coupling is exactly the same, compared to ab-initio results. The calculation of the MAE reveals that at theoretical equilibrium the easy axis is parallel to the wire, but at smaller interatomic distances corresponding to unsaturated magnetic solutions the easy axis is perpendicular to the wire. We have finally checked the validity of Bruno formula 8 relating the MAE to the anisotropy of the orbital moment, and found that this relation is almost strictly obeyed around the equilibrium distance. Indeed at this interatomic distance the up spin bands are filled and the exchange splitting is large compared to the $d$ bandwidth, which was not the case for the (001) surface that, although saturated, has much a wider $d$ bandwidth.

The paper is organized as follows. In section 2 the formalism of our model is presented in details, in particular the derivation of the $x, y$ and $z$ components of the orbital and spin moment formula in a non-orthogonal basis set. The spin-orbit coupling being small we have recalled the perturbation treatment of the MAE and of the orbital moment, from which the analytical expression of the anisotropy laws are directly obtained, and are used to analyze our numerical results. In section 3 the Stoner parameter is determined and used to study in detail the magnetic properties of bcc and fcc iron. Section 4 is devoted to the study of (001) and (110) surfaces. Finally in section 5 we present an exhaustive study of the monoatomic wire. Conclusions are drawn in section 6 .

\section{FORMALISM}

\section{A. Spin polarized tight-binding model}

We choose as a basis set the real $s, p$ and $d$ valence atomic orbitals centered on each site $i$. They are denoted by $\lambda$ and $\mu$ indices $(\lambda, \mu=1,9)$ and numbered as follows: $s, p_{x}, p_{y}, p_{z}, d_{x y}, d_{y z}, d_{z x}, d_{x^{2}-y^{2}}, d_{3 z^{2}-r^{2}}$, the $x, y, z$ coordinates being taken along the crystal axes. The tight-binding (TB) hamiltonian for the non-magnetic (NM) state is then completely determined by its intra-atomic matrix elements (i.e., the $s, p$ and $d$ atomic levels) $\varepsilon_{\lambda}$ and its interatomic matrix elements (i.e., the hopping integrals) $\beta_{i j}^{\lambda \mu}$ which have been tabulated as a function of 10 Slater-Koster 
(SK) parameters $(s s \sigma, s p \sigma, s d \sigma, p p \sigma, p p \pi, p d \sigma, p d \pi, d d \sigma, d d \pi, d d \delta)$ and of the direction cosines of the bonding direction $\mathbf{R}_{i j}$ 22]. Following the (MP) scheme developed by Mehl and Papaconstantopoulos [21], the atomic levels depend on the atomic environment (number of neighbors and interatomic distances) while the SK parameters are function of $R_{i j}$ only. Finally the Schroedinger equation in the atomic orbital basis involves also overlap integrals $\mathcal{S}_{i j}^{\lambda \mu}$ depending on the bonding direction $\boldsymbol{R}_{i j}$ when the non-orthogonality of the basis set is taken into account. All these quantities ( $\varepsilon_{\lambda}$, $\left.\beta_{i j}^{\lambda \mu}, \mathcal{S}_{i j}^{\lambda \mu}\right)$ are written as analytic functions depending on a number of parameters which are determined by a least mean square fit of the results of ab-initio electronic structure (band structure and total energy) calculations either in the Local Density (LDA) or in the Generalized Gradient (GGA) approximations. These parametrizations will be denoted as TBLDA and TBGGA in the following. The analytical form of the functions can be found in Ref. 21] and the numerical values of the parameters for Fe can be found in Ref. 23.

In order to account for spin polarization, we use a simplified Hartree-Fock (HF) scheme [1] to define atomic levels depending on spin $\left(\varepsilon_{\lambda \sigma}\right)$. These diagonal elements of the hamiltonian can be written in the basis of spin-orbitals $|i \lambda \sigma\rangle$ in which the spin quantization axis is parallel to the magnetization $(\sigma=+1(-1)$ for $\operatorname{up}($ down $)$ spin). When all atoms in the system are geometrically equivalent we get:

$$
\begin{aligned}
\varepsilon_{s, \sigma} & =\varepsilon_{0 s}+U_{s s} \frac{N_{s}}{2}+\left(U_{s p}-\frac{J_{s p}}{2}\right) N_{p}+\left(U_{s d}-\frac{J_{s d}}{2}\right) N_{d} \\
& -\frac{\sigma}{2}\left(U_{s s} M_{s}+J_{s p} M_{p}+J_{s d} M_{d}\right) \\
\varepsilon_{p_{\alpha} \sigma} & =\varepsilon_{0 p}+U_{s p} N_{s}+\left(U_{p p^{\prime}}-\frac{J_{p p^{\prime}}}{2}\right) N_{p}-\frac{1}{2}\left(U_{p p^{\prime}}-3 J_{p p^{\prime}}\right) n_{p_{\alpha}}+U_{p d} N_{d} \\
& -\frac{\sigma}{2}\left(J_{s p} M_{s}+J_{p p^{\prime}} M_{p}+J_{p d} M_{d}+\left(U_{p p^{\prime}}+J_{p p^{\prime}}\right) m_{p_{\alpha}}\right) \\
\varepsilon_{d_{\alpha} \sigma} & =\varepsilon_{0 d}+\left(U_{s d}-\frac{J_{s d}}{2}\right) N_{s}+\left(U_{p d}-\frac{J_{p d}}{2}\right) N_{p}+\left(U_{d d^{\prime}}-\frac{J_{d d^{\prime}}}{2}\right) N_{d}-\frac{1}{2}\left(U_{d d^{\prime}}-3 J_{d d^{\prime}}\right) n_{d_{\alpha}} \\
& -\frac{\sigma}{2}\left(J_{s d} M_{s}+J_{p d} M_{p}+J_{d d^{\prime}} M_{d}+\left(U_{d d^{\prime}}+J_{d d^{\prime}}\right) m_{d_{\alpha}}\right)
\end{aligned}
$$

where $N_{s(p, d)}$ and $M_{s(p, d)}$ are the total number of electrons and the total moment on each atom, respectively, in $s$, $p$ and $d$ orbitals while $n_{p_{\alpha}\left(d_{\alpha}\right)}$ and $m_{p_{\alpha}\left(d_{\alpha}\right)}$ denote the total occupation number (i.e., for both spins) and moment in orbital $p_{\alpha}\left(d_{\alpha}\right)$. Finally the $U$ and $J$ parameters are Coulomb and exchange integrals which involve two different orbitals, save for $U_{s s}$, and are assumed to depend only on the orbital quantum numbers of these orbitals.

We further assume that the asphericity of both the charge distribution and magnetic polarization can be neglected, i.e., $n_{p_{\alpha}}=N_{p} / 3, n_{d_{\alpha}}=N_{d} / 5, m_{p_{\alpha}}=M_{p} / 3, m_{d_{\alpha}}=M_{d} / 5$. In these conditions, when the system is non magnetic all the non vanishing terms in Eqs 1 are accounted for implicitly by the expression of $\varepsilon_{\lambda}$ in the MP scheme 21]. In addition the equations giving the atomic levels can be further simplified by noting that the spin polarization of $s$ and $p$ electrons is very small. As a consequence Eq.11 can be approximated by:

$$
\begin{aligned}
\varepsilon_{s, \sigma} & =\varepsilon_{s}-\frac{\sigma}{2} J_{s d} M_{d} \\
\varepsilon_{p, \sigma} & =\varepsilon_{p}-\frac{\sigma}{2} J_{p d} M_{d} \\
\varepsilon_{d, \sigma} & =\varepsilon_{d}-\frac{\sigma}{2} I_{d d^{\prime}} M_{d}
\end{aligned}
$$

where $\varepsilon_{s(p, d)}$ are the NM levels [21] and $I_{d d^{\prime}}=\left(U_{d d^{\prime}}+6 J_{d d^{\prime}}\right) / 5$ can be identified with the Stoner parameter. The numerical value of this parameter will be determined in section 3.1 in order to reproduce as closely as possible the 
variation of the magnetic moment as a function of the interatomic distance that can be obtained from an ab-initio calculation. Finally, $J_{s d}$ and $J_{p d}$ are one order of magnitude smaller than $I_{d d^{\prime}} \mathbb{\mathbb { 1 }}$ and we have taken $J_{s d}=J_{p d}=$ $I_{d d^{\prime}} / 10$. This completely defines our TB spin polarized hamiltonian $H_{T B H F}$ in the absence of spin-orbit coupling for a system of equivalent atoms, and when the overlaps are neglected.

In the general case where overlaps are taken into account and all atoms in the systems are not geometrically equivalent, the Hamiltonian becomes:

$$
H_{i j}^{\lambda \mu \sigma}=H_{i j}^{0, \lambda \mu}+\frac{U}{2}\left(\delta N_{i}+\delta N_{j}\right) \mathcal{S}_{i j}^{\lambda \mu}-\frac{\sigma}{4}\left(\Delta_{\lambda}^{i}+\Delta_{\mu}^{j}\right) \mathcal{S}_{i j}^{\lambda \mu}
$$

$H_{i j}^{0, \lambda \mu}$ are the matrix elements provided by the MP parametrization of the Hamiltonian. The second term in which $\delta N_{i}$ is the net total charge on atom $i$, prevents large charge transfers when inequivalent atoms are present, and will be discussed in section 4 . Finally in the last term which accounts for spin polarization $\Delta_{\lambda}^{i}=J_{s d} M_{d}^{i}, J_{p d} M_{d}^{i}$ and $I_{d d^{\prime}} M_{d}^{i}$ for $s, p, d$ orbitals, respectively.

\section{B. The spin-orbit coupling}

The spin-orbit interaction for a single atom is given by:

$$
H_{\mathrm{so}}=\frac{\hbar}{4 m^{2} c^{2}}(\nabla V \wedge \mathbf{p}) \cdot \boldsymbol{\sigma}
$$

where $V$ is the atomic potential, $\mathbf{p}$ is the momentum operator and $\boldsymbol{\sigma}$ are the Pauli matrices. Taking into account the spherical symmetry of the potential, $H_{\text {so }}$ can be rewritten as:

$$
H_{\mathrm{so}}=\xi(r) \mathbf{L} . \mathbf{S}
$$

with:

$$
\xi(r)=\frac{1}{2 m^{2} c^{2}} \frac{1}{r} \frac{d V}{d r}
$$

$\mathbf{L}=\mathbf{r} \wedge \mathbf{p}$ and $\mathbf{S}=\hbar \boldsymbol{\sigma} / 2$ are, respectively, the angular orbital and spin momentum operators. The matrix elements of $H_{\mathrm{so}}$ in the basis of atomic spin-orbitals $|\lambda \sigma\rangle$ are:

$$
\left\langle\lambda \sigma\left|H_{\mathrm{so}}\right| \mu \sigma^{\prime}\right\rangle=\xi_{\lambda \mu}\left\langle\bar{\lambda} \sigma|\mathbf{L} . \mathbf{S}| \bar{\mu} \sigma^{\prime}\right\rangle
$$

with:

$$
\xi_{\lambda \mu}=\int_{0}^{\infty} \mathcal{R}_{\lambda}(r) \mathcal{R}_{\mu}(r) \xi(r) r^{2} d r
$$


where $\mathcal{R}_{\lambda}(r)$ is the radial part of the atomic orbital $\lambda$ and $\bar{\lambda}$ denotes its angular part. Since $\xi(r)$ is well localized around $\mathbf{r}=\mathbf{0}, \xi_{\lambda \mu}$ has a non negligible value only when $\mathcal{R}_{\lambda}(r)$ and $\mathcal{R}_{\mu}(r)$ are also well localized, i.e., for transition metals, when both $\lambda$ and $\mu$ are $d$ orbitals in which case $\mathcal{R}_{\lambda}(r)=\mathcal{R}_{\mu}(r)=\mathcal{R}_{d}(r)$ and $\xi_{\lambda \mu}=\xi(\xi>0)$.

In the tight-binding approximation the crystal potential is written as $\mathcal{V}(\mathbf{r})=\sum_{i} V\left(\left|\mathbf{r}-\mathbf{R}_{i}\right|\right)$ and $H_{\text {so }}$ becomes:

$$
H_{\mathrm{so}}=\sum_{i} \xi\left(\left|\mathbf{r}-\mathbf{R}_{i}\right|\right) \mathbf{L}_{i} . \mathbf{S}
$$

where $\mathbf{L}_{i}$ is the angular orbital momentum operator with respect to the center $i$. For transition metals and due to the localized character of $\xi\left(\left|\mathbf{r}-\mathbf{R}_{i}\right|\right)$ we can neglect all matrix elements of $H_{\text {so }}$ save for the intra-atomic ones between $d$ orbitals. These matrix elements are the same at each site and given in Appendix A in the spin framework $x^{\prime \prime}, y^{\prime \prime}, z^{\prime \prime}$. In this framework $z^{\prime \prime}$ is the spin quantization axis defined by its polar and azimuthal angles $\theta, \varphi$ relative to the crystal axes. The $x^{\prime \prime}$ and $y^{\prime \prime}$ axes have been chosen in the following way: the $x, y$ axes of the crystal are first rotated by the angle $\varphi$ around $z$, this gives a new framework $x^{\prime}, y^{\prime}, z^{\prime}$ which is then rotated by an angle $\theta$ around $y^{\prime}$. The orbital and spin moments are usually expressed in units of $\hbar$ so that $\xi$ is a parameter which has the dimension of an energy. Its numerical value will be deduced from ab-initio calculations in the following.

\section{Determination of the components of the spin and orbital moments in the spin framework.}

\section{Spin moment.}

Let us first compute the average value of the three components of the total spin $<S_{x^{\prime \prime}}>,<S_{y^{\prime \prime}}>,<S_{z^{\prime \prime}}>$ in the spin framework. If we choose as a basis set of spin-orbitals the direct product of the orbitals $|i \lambda\rangle$ with the eigenvectors of the operator $S_{z^{\prime \prime}}$ denoted as $\uparrow$ and $\downarrow$, the electron eigenfunctions $\left|\psi_{n}\right\rangle$ in the crystal can be written:

$$
\left|\psi_{n}\right\rangle=\sum_{i \lambda} c_{i \lambda \uparrow}^{n}|i \lambda \uparrow\rangle+c_{i \lambda \downarrow}^{n}|i \lambda \downarrow\rangle=\sum_{i \lambda \sigma} c_{i \lambda \sigma}^{n}|i \lambda \sigma\rangle
$$

(Note that in the absence of spin-orbit coupling, there is no spin mixing in these eigenstates and, since the matrix elements of $H_{T B H F}$ are real, it is always possible to find a set of eigenvectors whose components are real and denoted as $c_{i \lambda \sigma}^{0 n}$ in the following). The average values of the three spin components are given by:

$$
\langle\mathbf{S}\rangle=\sum_{n \text { occ }}\left\langle\psi_{n}\left|\frac{\boldsymbol{\sigma}}{2}\right| \psi_{n}\right\rangle
$$

in a non orthogonal orbital basis set, we obtain:

$$
\begin{aligned}
& <S_{x^{\prime \prime}}>=\operatorname{Re} \sum_{\substack{i \lambda, j \mu \\
n \text { occ }}} c_{i \lambda \uparrow}^{n *} c_{j \mu \downarrow}^{n} \mathcal{S}_{i j}^{\lambda \mu} \\
& <S_{y^{\prime \prime}}>=\operatorname{Im} \sum_{\substack{i \lambda, j \mu \\
n \text { occ }}} c_{i \lambda \uparrow}^{n *} c_{j \mu \downarrow}^{n} \mathcal{S}_{i j}^{\lambda \mu} \\
& <S_{z^{\prime \prime}}>=\frac{1}{2} \sum_{\substack{i \lambda, j \mu \sigma \\
n \text { occ }}} \sigma c_{i \lambda \sigma}^{n *} c_{j \mu \sigma}^{n} \mathcal{S}_{i j}^{\lambda \mu}
\end{aligned}
$$


i.e., in the absence of spin-orbit coupling $<S_{x^{\prime \prime}}>=<S_{y^{\prime \prime}}>=0$.

For a system with full translational symmetry and a single atom per unit cell, the Bloch theorem yields $(\sigma=\uparrow$ or $\downarrow):$

$$
c_{i \lambda \sigma}^{n}=\frac{1}{\sqrt{N_{\mathrm{at}}}} \exp \left(i \mathbf{k} \cdot \mathbf{R}_{i}\right) c_{\lambda \sigma}^{\alpha}(\mathbf{k})
$$

since each eigenstate $n$ is labelled by a band index $(\alpha=1,9)$ and a wave vector $\mathbf{k} . N_{\text {at }}$ is the number of atoms. The spin components are the same on all sites $i$ and are given by:

$$
\begin{aligned}
&<S_{x^{\prime \prime}}>=\operatorname{Re} \sum_{\substack{\lambda \mu \\
(\alpha, \mathbf{k}) \text { occ }}} c_{\lambda \uparrow}^{\alpha *}(\mathbf{k}) c_{\mu \downarrow}^{\alpha}(\mathbf{k}) \mathcal{S}_{\lambda \mu}(\mathbf{k}) \\
&<S_{y^{\prime \prime}}>=\operatorname{Im} \sum_{\substack{\lambda \mu \\
(\alpha, \mathbf{k}) \text { occ }}} c_{\lambda \uparrow}^{\alpha *}(\mathbf{k}) c_{\mu \downarrow}^{\alpha}(\mathbf{k}) \mathcal{S}_{\lambda \mu}(\mathbf{k}) \\
&<S_{z^{\prime \prime}}>=\frac{1}{2} \sum_{\substack{\lambda \mu \sigma \\
(\alpha, \mathbf{k}) \text { occ }}} \sigma c_{\lambda \sigma}^{\alpha *}(\mathbf{k}) c_{\mu \sigma}^{\alpha}(\mathbf{k}) \mathcal{S}_{\lambda \mu}(\mathbf{k})
\end{aligned}
$$

with $\mathcal{S}_{\lambda \mu}(\mathbf{k})=N_{\text {at }}^{-1} \sum_{i j} \exp \left(i \mathbf{k} \cdot\left(\mathbf{R}_{j}-\mathbf{R}_{i}\right)\right) \mathcal{S}_{i j}^{\lambda \mu}$.

When all atoms are not geometrically equivalent, we can define a spin on site $i$ by identifying in Eqs.9 all the terms involving this site and, similarly to what is done to define Mulliken charges, the overlap cross terms (i.e., those in which only one of the site indices is equal to $i$ ) are multiplied by a factor $1 / 2$ to avoid a double counting of these terms. This condition ensures that these "local" spins are real and that their sum is equal to the total spin. For example in a periodic system with several atoms per unit cell the local spin on each atom in the cell are given by equations similar to equation 11 with an additional index, labelling the atom in the cell. For instance in a slab the local spin moment $<S_{a z}$ " $>$ on layer $a$ is given by:

$$
<S_{a z^{\prime \prime}}>=\frac{1}{4}\left(\sum_{\substack{b \lambda \mu \sigma \\\left(\alpha, \mathbf{k}_{\|}\right) \text {occ }}} \sigma\left(c_{a \lambda \sigma}^{\alpha *}\left(\mathbf{k}_{\|}\right) c_{b \mu \sigma}^{\alpha}\left(\mathbf{k}_{\|}\right) \mathcal{S}_{\lambda \mu}^{a b}\left(\mathbf{k}_{\|}\right)+c_{b \mu \sigma}^{\alpha *}\left(\mathbf{k}_{\|}\right) c_{a \lambda \sigma}^{\alpha}\left(\mathbf{k}_{\|}\right) \mathcal{S}_{\mu \lambda}^{b a}\left(\mathbf{k}_{\|}\right)\right)\right)
$$

with $\mathcal{S}_{\lambda \mu}^{a b}\left(\mathbf{k}_{\|}\right)=N_{\| \text {at }}^{-1} \sum_{\tilde{\mathfrak{1}} \mathrm{j}} \exp \left(i \mathbf{k}_{\| \cdot} \cdot\left(\mathbf{R}_{\tilde{\mathrm{J}}}-\mathbf{R}_{\tilde{\mathrm{I}}}\right)\right) \mathcal{S}_{\tilde{1} a \tilde{\jmath} b}^{\lambda \mu} . N_{\| \text {at }}$ is the number of atoms in each layer of the slab and $\mathbf{k}_{\|}$the wave vector parallel to the surface, each atom being now labelled by a cell index, $\tilde{\mathrm{i}}$ or $\tilde{\mathrm{j}}$, and a layer index, $a$ or $b$. Corresponding changes must be made for the two other components of $\mathbf{S}$. Finally let us recall that the spin magnetic moment $\mathbf{M}$ is related to the spin $\mathbf{S}$ by $<\mathbf{M}>=-2<\mathbf{S}>$ (in Bohr magnetons $\left.\mu_{B}\right)$.

\section{Orbital moment}

Up to now the orbital moment in the TB approximation has always been calculated by assuming an orthogonal basis set of atomic orbitals and only its $z^{\prime \prime}$ component was determined. In these conditions, the component of the 
local orbital moment on site $i$ in this direction is usually written as [17:

$$
<L_{i z^{\prime \prime}}>=\sum_{l m \sigma} m \int_{-\infty}^{E_{F}} \rho_{i l m \sigma}(E) d E
$$

where $\rho_{i l m \sigma}(E)$ is the local density of states at site $i$ projected on the atomic orbitals $|i l m\rangle=\mathcal{R}_{l}\left(r^{\prime \prime}\right) Y_{l m}\left(\theta^{\prime \prime}, \varphi^{\prime \prime}\right)$ and spin function $\sigma$, the variable $r^{\prime \prime}, \theta^{\prime \prime}, \varphi^{\prime \prime}$ being spherical coordinates relative to the spin framework, i.e.:

$$
\rho_{i l m \sigma}(E)=\sum_{\substack{l m \\ n}}\left\langle\psi_{n} \mid i l m \sigma\right\rangle\left\langle i l m \sigma \mid \psi_{n}\right\rangle \delta\left(E-E_{n}\right) .
$$

Thus

$$
\left\langle L_{i z^{\prime \prime}}\right\rangle=\sum_{\substack{\operatorname{lm} \sigma \\ n \text { occ }}}\left\langle\psi_{n} \mid i \lim \sigma\right\rangle m\left\langle i \lim \sigma \mid \psi_{n}\right\rangle
$$

This defines the operator $L_{i z^{\prime \prime}}$ which is diagonal in the $|i l m \sigma\rangle$ basis. Eq.15 can be generalized for the two other components of the orbital moment by noting that the corresponding operators are not diagonal in this basis. This gives:

$$
\left\langle\mathbf{L}_{i}^{\prime \prime}\right\rangle=\sum_{\substack{l m, l^{\prime} m^{\prime} \sigma \\ n \text { occ }}}\left\langle\psi_{n} \mid i l m \sigma\right\rangle\left[\mathbf{L}_{i}^{\prime \prime}\right]_{l m, l^{\prime} m^{\prime}}\left\langle i l^{\prime} m^{\prime} \sigma \mid \psi_{n}\right\rangle
$$

with $\mathbf{L}_{i}^{\prime \prime}=\left(L_{i x^{\prime \prime}}, L_{i y^{\prime \prime}}, L_{i z^{\prime \prime}}\right)$. Finally in the basis of real orbitals $|i \lambda \sigma\rangle$ defined in the crystal frame, we have:

$$
<\mathbf{L}_{i}^{\prime \prime}>=\sum_{\substack{\lambda \mu \sigma \\ n \text { occ }}} c_{i \lambda \sigma}^{n *}\left[\mathbf{L}_{i}^{\prime \prime}\right]_{\lambda \mu} c_{i \mu \sigma}^{n}
$$

The operators $\mathbf{L}_{i}^{\prime \prime}$ can be expressed as a function of the three operators $L_{i x}, L_{i y}, L_{i z}$ projecting the orbital moment on the crystal axes, i.e.:

$$
\begin{aligned}
& L_{i x^{\prime \prime}}=\cos \theta \cos \varphi L_{i x}+\cos \theta \sin \varphi L_{i y}-\sin \theta L_{i z} \\
& L_{i y^{\prime \prime}}=-\sin \varphi L_{i x}+\cos \varphi L_{i y} \\
& L_{i z^{\prime \prime}}=\sin \theta \cos \varphi L_{i x}+\sin \theta \sin \varphi L_{i y}+\cos \theta L_{i z}
\end{aligned}
$$

and the matrix elements of $\mathbf{L}_{i}$ between two atomic orbitals $\lambda$ and $\mu$ centered on atom $i$ defined with respect to the crystal axes are easily calculated (see Appendix A). These matrix elements are either vanishing or imaginary, thus $\left[\mathbf{L}_{i}\right]_{\lambda \mu}=-\left[\mathbf{L}_{i}\right]_{\mu \lambda}$. In the absence of spin-orbit coupling, as stated above, the coefficients $c_{i \lambda \sigma}^{0 n}$ are real and the orbital moment vanishes, i.e., $\left\langle\mathbf{L}_{i}^{\prime \prime}>=0\right.$.

Eq.17 can be generalized to take overlap into account (see Appendix B). This yields

$$
<\mathbf{L}_{i}^{\prime \prime}>=\operatorname{Re} \sum_{\substack{\lambda \mu j \nu \sigma \\ n \text { occ }}} c_{i \lambda \sigma}^{n *}\left[\mathbf{L}_{i}^{\prime \prime}\right]_{\lambda \mu} \mathcal{S}_{i j}^{\mu \nu} c_{j \nu \sigma}^{n}
$$


and, for a system with a full translational symmetry and a single atom per unit cell, this gives using Eq.10:

$$
<\mathbf{L}_{i}^{\prime \prime}>=\operatorname{Re} \sum_{\substack{\lambda \mu \nu \sigma \\ \alpha \mathbf{k} \text { occ }}} c_{\lambda \sigma}^{\alpha *}(\mathbf{k})\left[\mathbf{L}_{i}^{\prime \prime}\right]_{\lambda \mu} \mathcal{S}_{\mu \nu}(\mathbf{k}) c_{\nu \sigma}^{\alpha}(\mathbf{k})
$$

This latter equation can be easily generalized to periodic systems with several atoms per unit cell, in the same way as for the spin moment. Finally, let us note that in the presence of spin-orbit coupling, the direction of the total magnetization $\left(-<\mathbf{L}+2 \mathbf{S}>\right.$ ) may not strictly be parallel to the spin quantization axis $z^{\prime \prime}$. However $H_{\text {so }}$ being a small perturbation in Fe, we will often denote the spin quantization axis as the magnetization direction in the following.

\section{Magnetocrystalline anisotropy and orbital moment from perturbation theory}

We have seen in section 2.2 that spin-orbit effects can be limited to $d$ orbitals. Furthermore the overlaps between these orbitals are close to zero and the spin-orbit coupling is a weak perturbation since $\xi$ is much smaller than the Fe $d$ bandwidth. Consequently spin-orbit coupling effects can be understood using a simple perturbation theory with a basis set of orthogonal $d$ orbitals [6, 8, 24].

Let us consider the perturbation of the total energy due to $H_{\mathrm{so}}$. Since the matrix elements of $H_{\mathrm{so}}$ are a function of $\theta$ and $\varphi$, this introduces an angular dependence of this perturbation which is known as the magnetocrystalline anisotropy. The first order term can be written:

$$
\Delta E^{(1)}=\sum_{n \sigma \text { occ }}\left\langle n \sigma\left|H_{\mathrm{so}}\right| n \sigma\right\rangle
$$

where $|n \sigma\rangle$ is an unperturbed state of energy $E_{n \sigma}^{0}$, i.e.,

$$
|n \sigma\rangle=\sum_{i \lambda} c_{i \lambda \sigma}^{0 n}|i \lambda \sigma\rangle
$$

Thus:

$$
\Delta E^{(1)}=\xi \sum_{\lambda \mu}\langle\bar{\lambda} \sigma|\mathbf{L} . \mathbf{S}| \bar{\mu} \sigma\rangle \sum_{\substack{i \\ n \sigma_{\text {occ }}}} c_{i \lambda \sigma}^{0 n} c_{i \mu \sigma}^{0 n}
$$

It is easily seen that $\Delta E^{(1)}$ vanishes since for each spin the $(5 \times 5)$ matrix $\langle\bar{\lambda} \sigma|\mathbf{L} . \mathbf{S}| \bar{\mu} \sigma\rangle$ is imaginary (see appendix A).

The second order perturbation of the total energy is given by:

$$
\Delta E^{(2)}=-\sum_{\substack{n \sigma \text { occ } \\ n^{\prime} \sigma^{\prime} \text { unocc }}} \frac{\left|\left\langle n \sigma\left|H_{s o}\right| n^{\prime} \sigma^{\prime}\right\rangle\right|^{2}}{E_{n^{\prime} \sigma^{\prime}}^{0}-E_{n \sigma}^{0}} .
$$

This yields:

$$
\Delta E^{(2)}=-\xi^{2} \sum_{\lambda \mu \lambda^{\prime} \mu^{\prime}} \sum_{\sigma \sigma^{\prime}}\left\langle\bar{\lambda} \sigma|\mathbf{L} . \mathbf{S}| \bar{\mu} \sigma^{\prime}\right\rangle\left\langle\bar{\mu}^{\prime} \sigma^{\prime}|\mathbf{L} . \mathbf{S}| \bar{\lambda}^{\prime} \sigma\right\rangle \sum_{i j} I_{i j}\left(\lambda, \lambda^{\prime}, \mu^{\prime}, \mu, \sigma, \sigma^{\prime}\right)
$$


with:

$$
I_{i j}\left(\lambda, \lambda^{\prime}, \mu^{\prime}, \mu, \sigma, \sigma^{\prime}\right)=\int_{-\infty}^{E_{F}} d E \int_{E_{F}}^{\infty} d E^{\prime} \frac{\rho_{i j \sigma}^{0 \lambda \lambda^{\prime}}(E) \rho_{j i \sigma^{\prime}}^{0 \mu^{\prime} \mu}\left(E^{\prime}\right)}{E^{\prime}-E}
$$

and:

$$
\rho_{i j \sigma}^{0 \lambda \lambda^{\prime}}(E)=\sum_{n} c_{i \lambda \sigma}^{0 n} c_{j \lambda^{\prime} \sigma}^{0 n} \delta\left(E-E_{n \sigma}^{0}\right)
$$

By using the relations between the matrix elements of L.S shown by Bruno [6, 8] and recalled in Appendix A, $\Delta E^{(2)}$ can be rewritten as:

$$
\begin{aligned}
\Delta E^{(2)} & =\text { isotropic term } \\
& -\xi^{2} \sum_{\lambda \mu \lambda^{\prime} \mu^{\prime}}\langle\bar{\lambda} \uparrow|\mathbf{L} . \mathbf{S}| \bar{\mu} \uparrow\rangle\left\langle\overline{\mu^{\prime}} \uparrow|\mathbf{L} . \mathbf{S}| \overline{\lambda^{\prime}} \uparrow\right\rangle \sum_{i j, \sigma \sigma^{\prime}} \sigma \sigma^{\prime} I_{i j}\left(\lambda, \lambda^{\prime}, \mu^{\prime}, \mu, \sigma, \sigma^{\prime}\right) \\
& =\sum_{i} \Delta E_{i}^{(2)}
\end{aligned}
$$

where $\Delta E_{i}^{(2)}$ is the contribution of atom $i$ to the perturbation energy.

In the case of a system with full translational symmetry and a single atom per unit cell and using Eq.10, Eq.28 can be transformed into [6, 8]:

$$
\begin{aligned}
& \Delta E^{(2)}=\text { isotropic term }-\xi^{2} \sum_{\lambda \mu \lambda^{\prime} \mu^{\prime}}\langle\bar{\lambda} \uparrow|\mathbf{L} . \mathbf{S}| \bar{\mu} \uparrow\rangle\left\langle\overline{\mu^{\prime}} \uparrow|\mathbf{L} . \mathbf{S}| \overline{\lambda^{\prime}} \uparrow\right\rangle \\
& \quad \times \sum_{\mathbf{k}} \int_{-\infty}^{E_{F}} d E \int_{E_{F}}^{\infty} d E^{\prime} \frac{\mathcal{M}_{\lambda \lambda^{\prime}}(\mathbf{k}, E) \mathcal{M}_{\mu^{\prime} \mu}\left(\mathbf{k}, E^{\prime}\right)}{E^{\prime}-E}
\end{aligned}
$$

with:

$$
\mathcal{M}_{\lambda \lambda^{\prime}}(\mathbf{k}, E)=\mathcal{N}_{\lambda \lambda^{\prime} \uparrow}(\mathbf{k}, E)-\mathcal{N}_{\lambda \lambda^{\prime} \downarrow}(\mathbf{k}, E)
$$

and:

$$
\mathcal{N}_{\lambda \lambda^{\prime} \sigma}(\mathbf{k}, E)=\sum_{\alpha} c_{\lambda \sigma}^{0 \alpha *}(\mathbf{k}) c_{\lambda^{\prime} \sigma}^{0 \alpha}(\mathbf{k}) \delta\left(E-E_{\alpha \sigma}^{0}(\mathbf{k})\right)
$$

the superscript 0 refers to the unperturbed state as above and $E_{\alpha \sigma}^{0}(\mathbf{k})$ are the unperturbed eigenenergies.These equations can be generalized to a periodic system with several atoms per unit cell 11 .

Let us now consider the projection of the orbital moment on the spin framework axes. From Eq.17 it can be seen that the operators associated with these projections at a given site $i$ can be written in an orthogonal basis set:

$$
\mathbf{L}_{i}^{\prime \prime}=\sum_{\lambda \mu \sigma}|i \lambda \sigma\rangle\left[\mathbf{L}_{i}^{\prime \prime}\right]_{\lambda \mu}\langle i \mu \sigma|
$$


Within perturbation theory, we have:

$$
<\mathbf{L}_{i}^{\prime \prime}>=-\sum_{\substack{n \sigma \text { occ } \\ n^{\prime} \sigma^{\prime} \text { unocc }}} \frac{\left\langle n \sigma\left|\mathbf{L}_{i}^{\prime \prime}\right| n^{\prime} \sigma^{\prime}\right\rangle\left\langle n^{\prime} \sigma^{\prime}\left|H_{\mathrm{so}}\right| n \sigma\right\rangle}{E_{n^{\prime} \sigma^{\prime}}^{0}-E_{n \sigma}^{0}}+\text { c.c. }
$$

By substituting Eqs. 32 and 22 for $\mathbf{L}_{i}^{\prime \prime}$ and $|n \sigma\rangle$, respectively, into the preceding equation, we get:

$$
<\mathbf{L}_{i}^{\prime \prime}>=-2 \xi \sum_{\lambda \mu \lambda^{\prime} \mu^{\prime}} \sum_{\sigma}\left\langle\bar{\lambda} \sigma\left|\mathbf{L}_{i}^{\prime \prime}\right| \bar{\mu} \sigma\right\rangle\left\langle\overline{\mu^{\prime} \sigma}|\mathbf{L} . \mathbf{S}| \bar{\lambda}^{\prime} \sigma\right\rangle \sum_{j} I_{i j}\left(\lambda, \lambda^{\prime}, \mu^{\prime}, \mu, \sigma, \sigma\right)
$$

the factor 2 in Eq. 34 accounts for the complex conjugate in Eq.33 since the matrix elements of $\mathbf{L}_{i}^{\prime \prime}$ and $\mathbf{L} . \mathbf{S}$ for parallel spins are imaginary and all $I_{i j}$ are real. For a system we full translational symmetry and a single atom per unit cell this equation becomes [8]:

$$
\begin{array}{r}
\left\langle\mathbf{L}_{i}^{\prime \prime}>=-2 \xi \sum_{\lambda \mu \lambda^{\prime} \mu^{\prime}} \sum_{\sigma}\left\langle\bar{\lambda} \sigma\left|\mathbf{L}_{i}^{\prime \prime}\right| \bar{\mu} \sigma\right\rangle\left\langle\overline{\mu^{\prime} \sigma}|\mathbf{L} \cdot \mathbf{S}| \overline{\lambda^{\prime}} \sigma\right\rangle\right. \\
\quad \times \sum_{\mathbf{k}} \int_{-\infty}^{E_{F}} d E \int_{E_{F}}^{\infty} d E^{\prime} \frac{\mathcal{N}_{\lambda \lambda^{\prime} \sigma}(\mathbf{k}, E) \mathcal{N}_{\mu^{\prime} \mu \sigma}\left(\mathbf{k}, E^{\prime}\right)}{E^{\prime}-E}
\end{array}
$$

Furthermore noting that $\left[L_{i z^{\prime \prime}}\right]_{\lambda \mu}=2 \sigma\langle\bar{\lambda} \sigma|\mathbf{L} \cdot \mathbf{S}| \bar{\mu} \sigma\rangle$, Eq. 34 for $<L_{i z^{\prime \prime}}>$ can be transformed into:

$$
<L_{i z^{\prime \prime}}>=-4 \xi \sum_{\lambda \mu \lambda^{\prime} \mu^{\prime}} \sum_{\sigma} \sigma\langle\bar{\lambda} \uparrow|\mathbf{L} . \mathbf{S}| \bar{\mu} \uparrow\rangle\left\langle\overline{\mu^{\prime}} \uparrow|\mathbf{L} . \mathbf{S}| \overline{\lambda^{\prime}} \uparrow\right\rangle \sum_{j} I_{i j}\left(\lambda, \lambda^{\prime}, \mu^{\prime}, \mu, \sigma, \sigma\right)
$$

For a system with full translational symmetry and one atom per unit cell, this yields [8]

$$
\begin{aligned}
& <L_{i z^{\prime \prime}}>=-2 \xi \sum_{\lambda \mu \lambda^{\prime} \mu^{\prime}}\langle\bar{\lambda} \uparrow|\mathbf{L} \cdot \mathbf{S}| \bar{\mu} \uparrow\rangle\left\langle\overline{\mu^{\prime}} \uparrow|\mathbf{L} \cdot \mathbf{S}| \overline{\lambda^{\prime}} \uparrow\right\rangle \\
& \quad \times \sum_{\mathbf{k}} \int_{-\infty}^{E_{F}} d E \int_{E_{F}}^{\infty} d E^{\prime} \frac{\mathcal{N}_{\lambda \lambda^{\prime}}(\mathbf{k}, E) \mathcal{M}_{\mu^{\prime} \mu}\left(\mathbf{k}, E^{\prime}\right)+\mathcal{M}_{\lambda \lambda^{\prime}}(\mathbf{k}, E) \mathcal{N}_{\mu^{\prime} \mu}\left(\mathbf{k}, E^{\prime}\right)}{E^{\prime}-E}
\end{aligned}
$$

in which $\mathcal{N}_{\lambda \lambda^{\prime}}(\mathbf{k}, E)=\sum_{\sigma} \mathcal{N}_{\lambda \lambda^{\prime} \sigma}(\mathbf{k}, E)$. The generalization of this equation to systems with several atoms per unit cell is straightforward.

It can be seen from Eqs 28 and 36 that $\left\langle L_{i z^{\prime \prime}}>\right.$ and the anisotropic part of $\Delta E_{i}^{(2)}$ are both given by quadratic functions of the direction cosines of the spin quantization axis relative to the crystal ones since the involved matrix elements of L.S are all proportional to one of these direction cosines (see Appendix A). These two functions present some similarity, but spin-flip excitations contribute to $\Delta E_{i}^{(2)}$ but not to $\left\langle L_{i z^{\prime \prime}}\right\rangle$. However, if the exchange splitting is large enough compared to the $d$ bandwidth, the spin up band is completely filled and the contribution of spin-flip excitations to $\Delta E_{i}^{(2)}$ is negligible due to the large value of the energy denominator. In this condition, for each site $i$, the anisotropy of $\Delta E_{i}^{(2)}$ and $<L_{i z^{\prime \prime}}>$ are proportional:

$$
\Delta E_{i}^{(2)}(\theta, \varphi)-\Delta E_{i}^{(2)}(0,0)=-\frac{\xi}{4}\left(<L_{i z^{\prime \prime}}(\theta, \varphi)>-<L_{i z^{\prime \prime}}(0,0)>\right)
$$


Note that this relation was already derived by Bruno [6] for fcc monolayers with a single atom per unit cell. Finally let us point out that for bulk cubic crystals with a single atom per unit cell both $\Delta E^{(2)}$ and $<L_{i z^{\prime \prime}}>$ are isotropic at the orders of perturbation considered.

\section{E. The ab-initio method}

For the sake of comparison we have also performed spin-polarized ab-initio calculations based on the Density Functional Theory (DFT) using the PWscf code of $\nu$-ESPRESSO package 25 with ultrasoft pseudopotentials including non-linear core corrections. The calculations without spin-orbit coupling have been carried out within the GGA and the Perdew-Wang exchange-correlation parametrization, while the one including spin-orbit coupling have been performed within the LDA and the Perdew-Zunger exchange-correlation parametrization. The plane wave kinetic-energy cut-off was taken equal to $35 \mathrm{Ry}$ for the wavefunctions and $250 \mathrm{Ry}$ for the charge density and potential, which ensures a very good energy precision.

\section{F. Computational details}

When dealing with magnetic properties, and in particular magnetic anisotropy, the convergence of the total energy with respect to the number of $k$-points has to be checked carefully. For all calculations involving magnetic anisotropy we checked that our results did not change by more than a few hundredth of meV (at most 0.1 meV in the worst case). In the case of PWscf calculations the use of plane waves imposes a periodically repeated geometry and one must also avoid as much as possible electronic interactions by using large unit cells. The monatomic wires were separated by 30a.u., but for surfaces, we have been less demanding since we only calculated the magnetic moment and therefore the slabs were separated by approximately 17a.u.

\section{BULK MAGNETISM OF BCC AND FCC IRON}

\section{A. Determination of the Stoner parameter $\mathbf{I}_{d d^{\prime}}$ from the magnetic transition in bcc iron}

In our TBHF model, the magnetism is entirely governed by the value of the Stoner parameter $I_{d d^{\prime}}$. It is well known that, in unsaturated magnetic materials like Fe, the magnetic moment is very sensitive to the precise value of the equilibrium interatomic distance. As a general trend, an expansion of the bulk lattice parameter leads to narrower (thus higher) density of states, which usually plays in favor of magnetism: it increases the magnetization in magnetic materials or it can trigger a magnetic transition in non-magnetic materials. Therefore a straightforward way to determine $I_{d d^{\prime}}$ is to study the evolution of the magnetic moment as a function of the lattice parameter. In Fig. 1 the result of a series of TBLDA and TBGGA calculations on bulk bcc iron is shown for various values of the Stoner parameter. As expected the magnetic moment increases when the lattice is expanded but also when the Stoner parameter is increased. With $I_{d d^{\prime}}=1 \mathrm{eV}$ (TBLDA) and $I_{d d^{\prime}}=1.10 \mathrm{eV}$ (TBGGA) we have been able to reproduce closely the results of PWscf calculations in a range of Wigner-Seitz radii $\left(R_{W S}\right)$ around equilibrium (the experimental bcc lattice parameter, $2.87 \AA$, corresponds to $R_{W S}=2.67$ a.u.). In the following we will keep these values fixed and 
neglect any variation of these parameters with the local atomic environment. Let us however note that at very large lattice spacings the spin moment saturates (not seen in Fig.1) at an "atomic" value of $2 \mu_{B}$ for TBLDA and $4 \mu_{B}$ for TBGGA. These two limits correspond to the different atomic configurations $3 d^{8} 4 s^{0}$ and $3 d^{6} 4 s^{2}$ found in TBLDA and TBGGA, respectively, for a free Fe atom. Therefore the TBLDA gives a wrong atomic configuration which will have some consequences on the surface magnetism. Let us finally mention that in ab-initio calculations LDA and GGA yield very similar results as far as the magnetic moment is concerned.

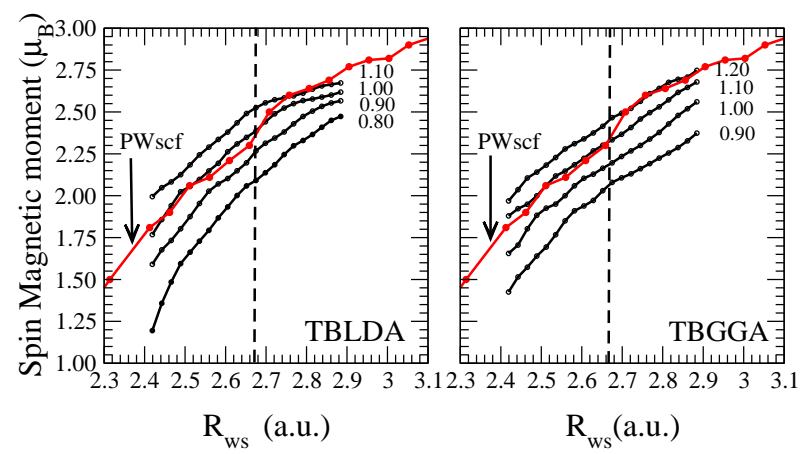

FIG. 1: Variation of the absolute value of the spin magnetic moment (per atom) of bcc Fe as a function of the Wigner-Seitz radius $R_{W S}$ for the values of the Stoner parameter $I_{d d^{\prime}}$ (in $\mathrm{eV}$ ) given on each curve. Left and right panels correspond to TBLDA and TBGGA calculations, respectively, compared to PWscf calculations in GGA. The dashed vertical line gives the experimental Wigner-Seitz radius at equilibrium.

\section{B. Fcc iron}

The ground state phase of iron in normal temperature and pressure conditions is ferromagnetic (FM) bcc, at higher temperatures, the fcc phase is stabilized but in a NM configuration. However it has been shown experimentally that thin films of iron can be stabilized in an fcc structure [26, 27]. This experimental work also showed the existence of various magnetic phases. It has also been known for a long time from theoretical works [28, 29, 30, 31, 32] that fcc iron has a much more complicated magnetic structure than bcc iron. In the following, we present a study of the magnetic properties of fcc Fe with TBGGA parameters. This will provide us with a first check of our model.

\section{Magnetic transition in fcc iron.}

We have carried out a series of calculations on the fcc phase of iron. Fig. 22 is similar to Fig. 11 but for the FM (a) and antiferromagnetic (AFM)(b) bulk fcc phases, the latter corresponding to a stacking of (001) planes in which spins of adjacent layers are opposite. In the FM case (Fig. 2a), the curves of appearance of a magnetic moment show a strong dependence on the magnetic moment $M_{0}$ chosen as input to begin the self-consistency iterations (a similar behavior is also found with the PWscf code). For large $M_{0}$ an abrupt transition from a NM configuration to a High Spin (HS) state occurs, for instance at $R_{W S} \simeq 2.6 \mathrm{a}$.u. when $M_{0}=3 \mu_{B}$. For a small value of $M_{0}$ a similar transition appears but at a much larger volume $\left(R_{W S} \simeq 2.7 \mathrm{a} . \mathrm{u}\right)$, while for intermediate $M_{0}$ this transition is less steep. 
The magnetic transition is much less abrupt in the antiferromagnetic phase (Fig.2 b b were two steps are observed: first, from a NM state to a low spin state (LS) and, second, from the LS state to the HS state. Note that a similar dependence on the input magnetic moment also exists in the AFM state (not shown on the graph).

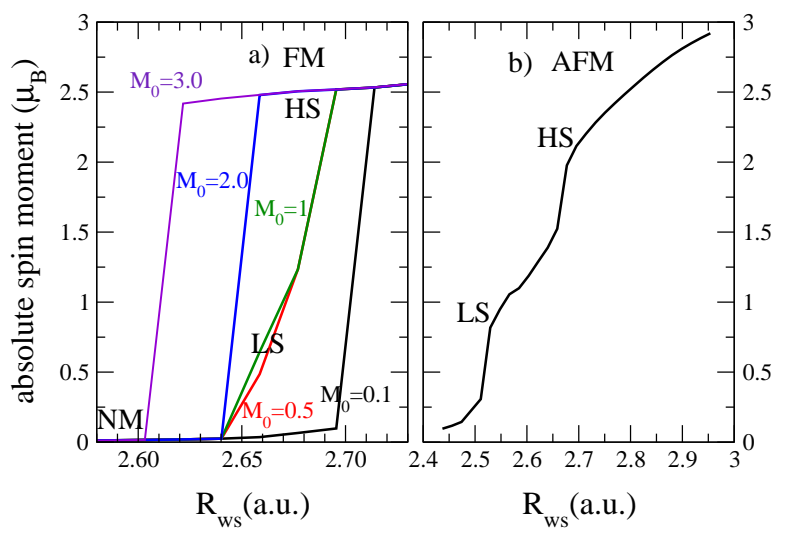

FIG. 2: Variation of the absolute value of the magnetic moment (per atom) for the ferromagnetic (FM) and antiferromagnetic (AFM) states of fcc Fe as a function of the Wigner-Seitz radius $R_{W S}$, for various input magnetic moments $M_{0}$ in $\mu_{B}$ obtained with TBGGA parameters. LS and HS denote respectively low and high spin states.

2. Low and High spin ferromagnetic states: fixed spin moment calculations.

The strong dependence on the input magnetic moment suggests the existence of metastable magnetic solutions. Therefore we have carried out TBGGA calculations using a fixed spin moment procedure for a series of Wigner-Seitz radii corresponding to the region of the magnetic transition. The behavior of the total energy as a function of the total moment M (Fig. 3) reveals the existence of several local minima. In particular the curve at $R_{W S}=2.67$ a.u. exhibits three minima (inset of Fig.3): one at $M=0$, one around $M=1.2 \mu_{B}$ and one at $M=2.5 \mu_{B}$, corresponding to the NM, LS and HS states, respectively. This complex energy behavior is in agreement with the results obtained by Moruzzi et al. 33] who showed for the first time the existence of three phases. It is clear that depending on the value of the input moment, the iteration loop will converge towards one of the three self-consistent (stable or metastable) magnetic states.

\section{Relative phase stability: comparison between TBLDA and TBGGA models.}

It is well known that DFT in the LDA predicts that, at low temperature, the fcc AFM phase is the most stable one contrary to experiments [34]. However the right phase stability is recovered with the GGA. It is therefore interesting to investigate the ground state properties of bulk iron within our TB model. The results are presented in Fig. 1 , it is found that TBLDA, similarly to DFT-LDA, gives the AFM state of fcc iron as the most stable phase, while with TBGGA the FM bcc phase is found to be the ground state. These results are in perfect agreement with ab-initio findings, showing the ability of our model to reproduce rather complex magnetic behaviors. 


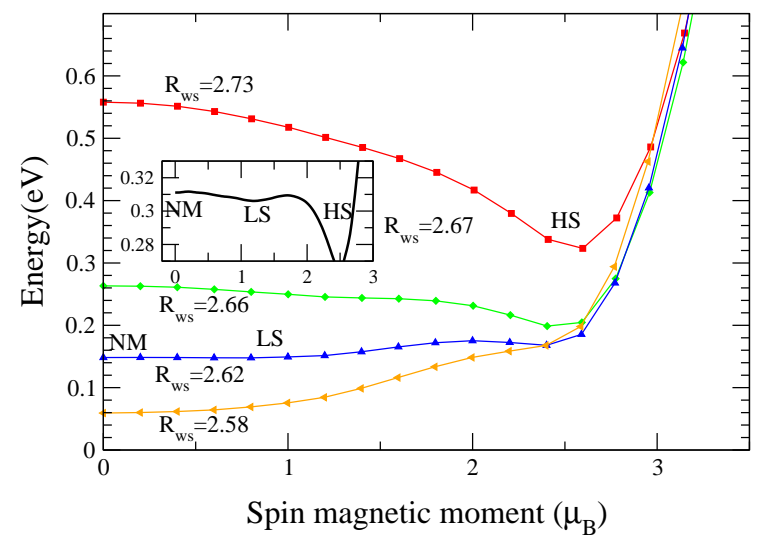

FIG. 3: Variation of the TBGGA total energy per atom with the magnetic moment (fixed spin moment calculation for the ferromagnetic state) for fcc Fe at several Wigner-Seitz radii $R_{W S}$ in a.u.. Note the presence of stable (or metastable) non magnetic (NM), Low spin (LS) and High spin (HS) states which is clearly seen in the inset. The zero of energy is arbitrary.

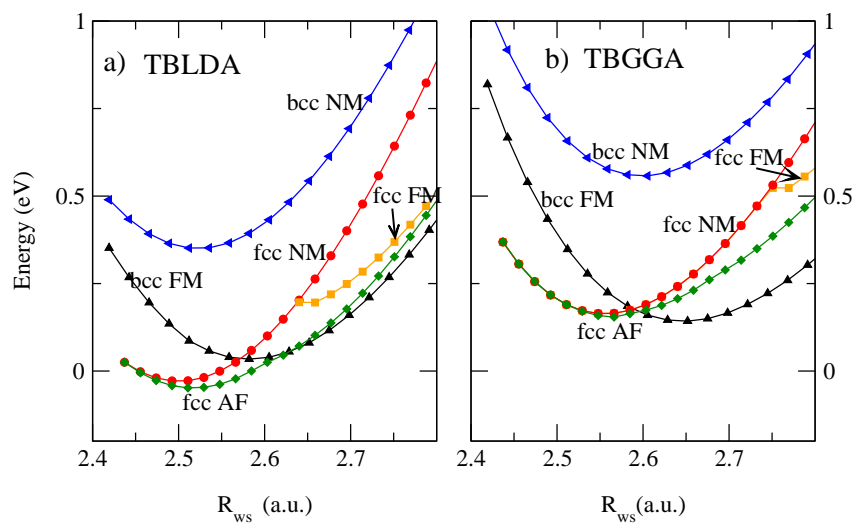

FIG. 4: Total energy per atom as a function of the Wigner Seitz radius $R_{W S}$ for ferromagnetic (FM), antiferromagnetic (AFM) and non magnetic (NM) states of bcc and fcc iron. Panels a) and b) correspond to calculations performed with TBLDA and TBGGA, respectively. The zero of energy is arbitrary but is the same for all the curves in each panel.

\section{Influence of spin-orbit coupling}

The results presented above have been obtained without including spin-orbit coupling effects. The value of the spinorbit coupling parameter $\xi$ can be deduced from a comparison of the NM bcc band structure along a high symmetry direction of the Brillouin zone, for instance $\Gamma H$, obtained with our model and with the PWscf code. Indeed, the effect of spin-orbit coupling is to remove the degeneracy of degenerate levels when a matrix element of $H_{\text {so }}$ exists between the corresponding eigenstates (see Appendix A). For instance it is easily seen that the six-fold degenerate level $\Gamma_{25^{\prime}}^{0}$, corresponding to $t_{2 g}$ spin-orbitals, are coupled by some matrix elements of $H_{\mathrm{so}}$. Using the perturbation theory for degenerate levels, it is easily found that this level splits into a four-fold degenerate level at $\Gamma_{25^{\prime}}^{0}-\xi / 2$ and a doubly degenerate level at $\Gamma_{25^{\prime}}^{0}+\xi$. From this splitting calculated with the PWscf code, we obtain $\xi=0.06 \mathrm{eV}$. We have verified that with this value, our model is able to reproduce perfectly the spin-orbit coupling effects along $\Gamma H$.

As seen in section 2, spin-orbit coupling is at the origin of the magneto-crystalline anisotropy. However, since this 
anisotropy is of fourth order in $\xi$, typical values for bulk materials are very small and of the order of $10^{-5}-10^{-6} \mathrm{eV}$ per atom for $\mathrm{Fe}$, Co or $\mathrm{Ni}$ which makes the calculation of this anisotropy almost impossible, since it is beyond the accuracy of electronic structure methods. On the contrary, reliable values of the orbital moment, which is isotropic in the bulk to first order in perturbation, can be derived from Eq.20. With our model and TBGGA parameters we find $\left\langle L_{z}\right\rangle=0.07 \mu_{B}$ in good agreement with experiments $\left(0.08 \mu_{B}[35)\right.$.

As a conclusion, the TB results presented above (section 3.2 and 3.3) are in perfect agreement with DFT calculations, showing the ability of our model to reproduce rather complex magnetic behaviors.

\section{IV. (110) AND (001) SURFACES OF IRON}

We have then applied our TBGGA model to the study of the (001) and (110) surfaces of bcc iron. At the surface some atoms have a reduced coordination and therefore charge transfers as large as some tenths of electron are found in our model if the atomic levels $\varepsilon_{s}, \varepsilon_{p}, \varepsilon_{d}$ in Eq.2 2 are kept at the values given by the MP equations. However, it is known that in metals, due to screening, the charge transfers are expected to be at least one order of magnitude smaller. To avoid unphysical charge transfers at surfaces the Hamiltonian is corrected by adding a term depending on the charge transfer $\delta N_{i}$ and of an average Coulomb integral $U$ which must be large enough $(U=5 \mathrm{eV})$ as shown in Eq.3.

\section{A. Band structure of the (110) surface}

In a previous work on rhodium surfaces we showed that the charge quasi-neutrality is crucial to obtain a good description of the surface and resonant states[36]. Indeed these states are extremely sensitive to the energy shift induced by the renormalization of the intra-atomic terms of the TB Hamiltonian. Here we have carried out a TBGGA projected band structure calculation for the (110) surface of bcc Fe. The results are shown in Fig. 5. It can be seen that the position and size of pseudo-gaps in the band structure is significantly different for up and down spins. In particular along the $\bar{\Gamma} \bar{S}$ and $\bar{S} \bar{H}$ directions the pseudo-gaps are much larger in the minority spin band structure than in the majority spin one. As a consequence there are more minority spin than majority spin surface states. This is evidenced by the presence of a sharp down spin surface state around the Fermi level (indicated by an arrow in Fig.

5) which disappears in the up spin band structure. These results are in excellent agreement with previous ab-initio calculations [37 in particular, for the position and dispersion of the characteristic down spin surface state discussed above.

\section{B. Spin magnetic moments of $\mathrm{Fe}(110)$ and $\mathrm{Fe}(001)$ surfaces}

It is well known that the lowering of coordination induces a narrowing of the density of states which usually enhances the magnetic moment. Consequently, it is expected that open surfaces should have larger surface magnetic moments than close-packed ones. We have therefore carried out self consistent TBGGA and PWscf GGA calculations for (001) and (110) surfaces. The (001) surface being more open than the (110) one, since each atom from their outermost layer looses 4 and 2 first nearest neighbors, respectively, we expect larger surface magnetic moments for the (001) 

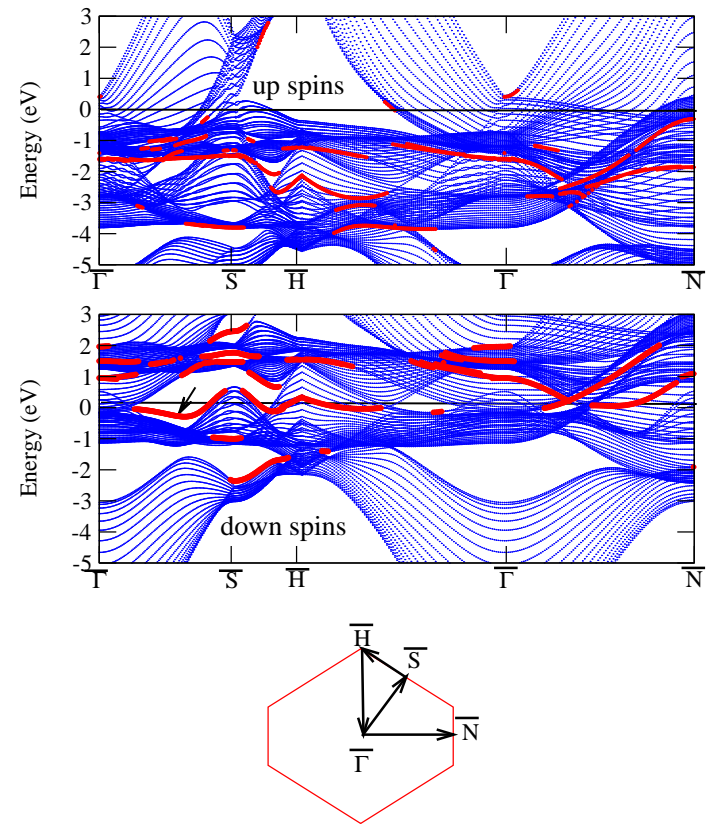

FIG. 5: TBGGA projected band structure for up (top) and down (middle) spins of a 20-layer (110) slab of bcc Fe with the experimental lattice parameter of $2.87 \AA$. The energy zero is the Fermi level. Surface or resonant states (i.e., states with more than $60 \%$ of their total weight on the first two outer layers) are represented in red and with thicker dots. A characteristic surface state of minority spin is indicated by an arrow. A schematic representation of the surface Brillouin zone and of the path in the reciprocal space is shown at the bottom.

than for the (110) surface. Fig. 6 shows that this general rule of thumb is well obeyed. Actually two clear features are seen in Fig. 6: the magnetic moment is more reinforced on the (001) than on the (110) surface $(+38 \%$ and $+14 \%$, respectively, for the outermost layer compared to the bulk ). Friedel type oscillations are present on the (001) surface while an almost monotonic decrease is obtained for the (110) surface. An excellent agreement is once again observed between PWscf and TBGGA results, in particular the spin moment is almost saturated on the outermost layer of the (001) surface in both calculations. Let us however note that the agreement is less perfect within TBLDA (not shown), which can be attributed to the wrong atomic configuration obtained in this model which deteriorates the $s p d$ charge distribution on the surface plane.

\section{Magneto-crystalline anisotropy}

For surfaces the magneto-crystalline anisotropy is usually one or two orders of magnitude larger than in the bulk. Indeed, it is well known that, contrary to the bulk, this anisotropy is of the second order in $\xi$ at surfaces. Actually, we have seen in section 2.4 that second order perturbation theory predicts that the magneto-crystalline anisotropy is a quadratic function of the direction $\operatorname{cosines}(l=\sin \theta \cos \varphi, m=\sin \theta \sin \varphi, n=\cos \theta)$ of the spin quantization axis relative to crystal axes. By imposing the symmetry properties of the surface to this quadratic form the following laws 


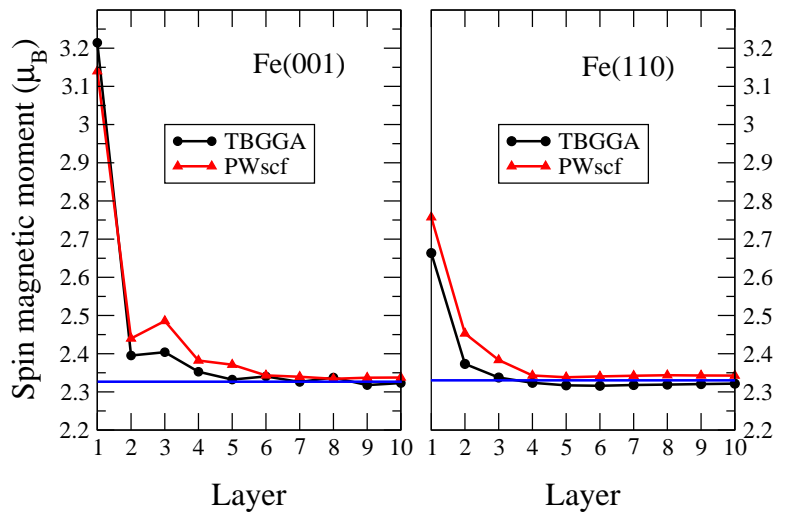

FIG. 6: Variation of the spin magnetic moment (per atom) on successive atomic layers of (001) and (110) slabs (with 20 atomic layers) of bcc Fe obtained from the TBGGA model and PWscf code with GGA. Layer 1 corresponds to the outermost layer and layer 10 to a central layer. The value of the bulk magnetic moment is indicated as a reference.

are easily derived:

$$
\Delta E_{i}^{(2)}(\theta, \varphi)-\Delta E_{i}^{(2)}(0,0)=K_{1}^{(001)} \sin ^{2} \theta
$$

for the (001) surface with $x$ and $y$ crystal axes parallel to the edges of the square two dimensional cell, and:

$$
\Delta E_{i}^{(2)}(\theta, \varphi)-\Delta E_{i}^{(2)}(0,0)=K_{1}^{(110)} \sin ^{2} \theta+K_{2}^{(110)} \sin ^{2} \theta \cos 2 \varphi
$$

on the (110) surface. For this surface the crystal axes are chosen as follows: the $z$ axis is perpendicular to the surface and the $y$ one is parallel to the second nearest neighbor direction in the surface.

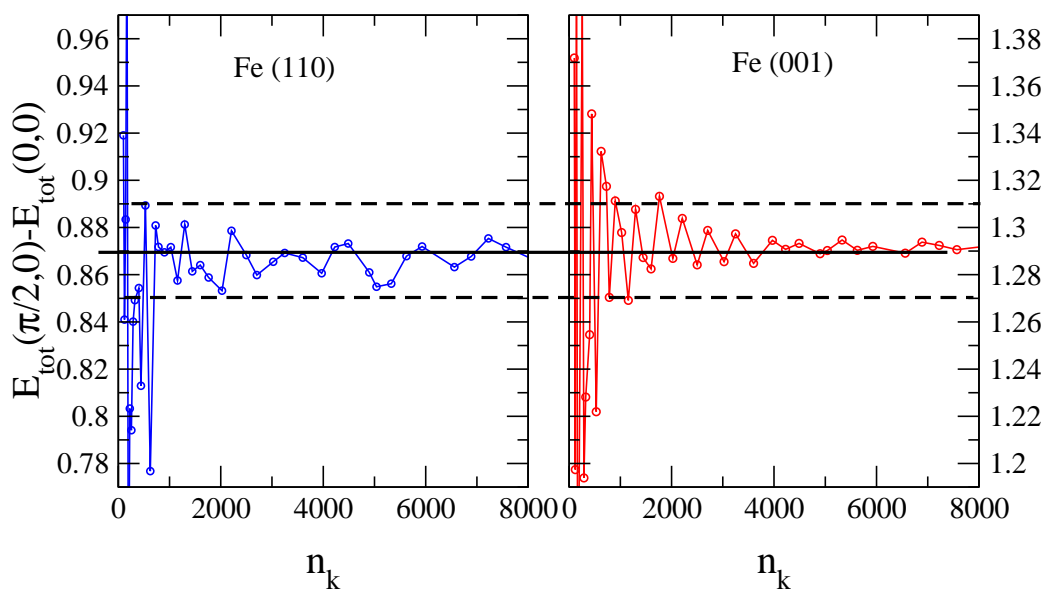

FIG. 7: Convergence of the magnetic anisotropy $E_{t o t}(\pi / 2,0)-E_{t o t}(0,0)$ with respect to the number of $k$ points $n_{k}$ in the first Brillouin zone, for (110) and (001) Fe bcc, unsupported monolayers. The magnetic anisotropy is oscillating around its asymptotic value (full straight line) with an amplitude below $\pm 0.02 \mathrm{meV}$ when $n_{k}$ is larger than 1000 . 


\begin{tabular}{|c|c|c|c|c|}
\hline Surface & $n_{l}$ & $n_{k}$ & $E_{\text {tot }}(\pi / 2,0)-E_{\text {tot }}(0,0)$ & $E_{\text {tot }}(\pi / 2, \pi / 4)-E_{\text {tot }}(\pi / 2,0)$ \\
\hline$(001)$ & 1 & 40000 & $1.29 \mathrm{meV}$ & $0.049 \mathrm{meV}$ \\
\hline$(001)$ & 11 & 1024 & $0.45 \mathrm{meV}$ & $0.041 \mathrm{meV}$ \\
\hline \hline & & & $E_{\text {tot }}(\pi / 2,0)-E_{\text {tot }}(0,0)$ & $E_{\text {tot }}(\pi / 2, \pi / 2)-E_{\text {tot }}(\pi / 2,0)$ \\
\hline$(110)$ & 1 & 40000 & $0.86 \mathrm{meV}$ & $-0.087 \mathrm{meV}$ \\
\hline$(110)$ & 11 & 1024 & $0.19 \mathrm{meV}$ & $-0.17 \mathrm{meV}$ \\
\hline
\end{tabular}

TABLE I: Magneto-crystalline anisotropy, $E_{t o t}(\theta, \varphi)$ being the total energy (per surface unit cell) corresponding to a magnetization direction defined by the angles $\theta, \varphi$ with respect to the crystal axes (see text) for slabs of bcc Fe with (001) and (110) orientations: $n_{l}$ is the number of layers and $n_{k}$ is the number of $k$ points in the first Brillouin zone used in the calculation.

In Table I we present the results of TBGGA calculations on (001) and (110) slabs of two thicknesses. We have first checked the convergence of the magnetic anisotropy with respect to the number of $k$-points in the first Brillouin zone. It is seen in Fig. 7 that a good convergence is obtained above $1000 k$-points. It is found that for both orientations the easy axis is perpendicular to the surface plane. The two surfaces, however, have a different behavior with respect to inplane magnetization. For the (001) surface the energy is changing by only $4.10^{-5} \mathrm{eV}$ between a magnetic configuration along the square edge $(\varphi=0)$ and along the diagonal of the square $(\varphi=\pi / 4)$. This confirms our previous symmetry analysis which predicts no in-plane dependence of the energy at second order in perturbation. In the case of the (110) slab with 11 layers the in-plane energy variation is almost one order of magnitude larger and plays in favor of the second nearest neighbor atomic direction $(\varphi=\pi / 2)$. Consequently the anisotropy is very small in the $y z$ plane. Let us also point out that isolated monolayers show a more pronounced out of plane anisotropy while the in-plane energy profile seems to be less corrugated than for thicker layers in the case of the (110) orientation.

\section{Orbital moment}

In presence of a surface the coordination is reduced and the symmetry is lowered leading to an enhancement of the spin moments as discussed above. At surfaces the orbital moment is also enhanced as demonstrated in several theoretical and experimental [38, 39] works. Our calculations on (001) and (110) slabs show that the component $<L_{i z^{\prime \prime}}>$ of the orbital moment on the magnetization direction is noticeably increased when $i$ belongs to the outermost layer, especially for the (001) surface. On the second layer this component has almost recovered its bulk value for the (110) surface whereas oscillations occur for the (001) surface similarly to the behavior of the spin moment. Finally $<L_{i z^{\prime \prime}}>$, contrary to the spin moment, depends sensitively on the direction of the magnetization with the same type of laws (Eqs. 39 and 40) as the magneto-crystalline anisotropy. However it is found that for $\theta=\pi / 2$ the $\varphi$ dependence predicted by Eq.40 for the (110) surface is almost negligible. On the contrary the variation of $<L_{i z^{\prime \prime}}>$ with $\theta$ is noticeable and, in this respect, the two surfaces behave differently: when the magnetization direction is rotated from $\theta=0$ to $\theta=\pi / 2,<L_{i z^{\prime \prime}}>$ decreases for the (001) surface while it increases for the (110) one. In addition, this variation is larger in absolute value on the (001) than on the (110) surface.

Finally it is expected that equation 38 should be more obeyed for (001) surface than for the (110) since the spin moment at the outermost plane is saturated for the former and not for the latter. Assuming that the contribution to the magnetocrystalline energy comes from the outermost plane only, the ratio of the magnetocrystalline anisotropy 
to that of the orbital moment for surface atoms, has the wrong sign for (110), while for the (001) surface the sign is correct but the ratio is around three times smaller than $\xi / 4$. Indeed the exchange splitting of the (001) surface is smaller than the $d$ bandwidth and the contribution of spin-flip excitations to the magnetocrystalline anisotropy cannot be neglected.

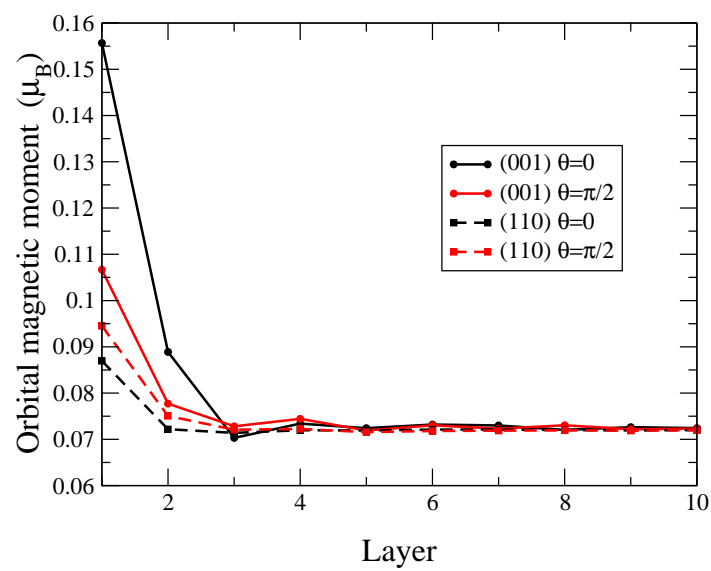

FIG. 8: Variation of the component of the orbital magnetic moment on the magnetization direction (per atom) as a function of the atomic layer in the (110) and (001) slabs (20 layers) for a magnetization perpendicular $(\theta=0)$ or parallel to the surface $(\theta=\pi / 2, \varphi=0)$.

\section{STUDY OF THE MONATOMIC WIRE}

Although the study of the unsupported monatomic wire, i.e., a periodic linear chain of identical atoms with a single atom per unit cell, is somewhat academic it is however an interesting object for the following reasons: i) it can be used as a model since analytical TB results can be derived which are useful for a theoretical understanding and a direct identification of the orbital character of the bands obtained in ab-initio calculations, ii) it also allows to investigate how a reduced dimensionality may modify magnetism in ferromagnetic metals 40 or induce it in nonmagnetic materials 41, 42, 43, iii) last, but not least, such objects, several atom long, have been observed in break junction experiments 44 (unfortunately not for $\mathrm{Fe}, \mathrm{Co}$ or $\mathrm{Ni}$ ).

\section{A. Non magnetic band structure of the monatomic wire}

The band structure of the non magnetic Fe monatomic wire, neglecting spin-orbit coupling, obtained from the TBLDA model and from the ab-initio PWscf code in the GGA are shown in Fig. 9 for an interatomic distance of 4.29

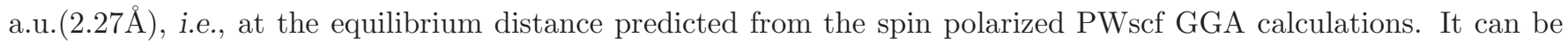
seen that except for the upper band in the PWscf band structure, the agreement is satisfactory.

Let us now identify the character of the bands by using the simplest TB model, i.e., in which overlap is neglected and hopping integrals are restricted to first nearest neighbors. We take the $z$ axis along the chain. For a given spin the $(9 \times 9)$ hopping matrix can be rearranged into five square blocks on the diagonal: the first one involves $s, p_{z}$ and 
$d_{3 z^{2}-r^{2}}$ orbitals, the second and third ones are identical and involve $\left(p_{x}, d_{z x}\right)$ and $\left(p_{y}, d_{y z}\right)$ orbitals, respectively, finally the fourth and fifth ones are also identical and correspond to the $d_{x y}$ and $d_{x^{2}-y^{2}}$ orbitals, respectively. Consequently, taking into account spin degeneracy, the band structure consists in three two-fold degenerate bands of symmetry $\sigma$, two four-fold degenerate bands of symmetry $\pi$ and one four-fold degenerate band of symmetry $\delta$.

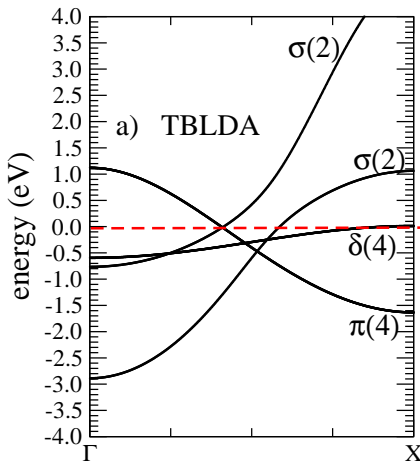

k

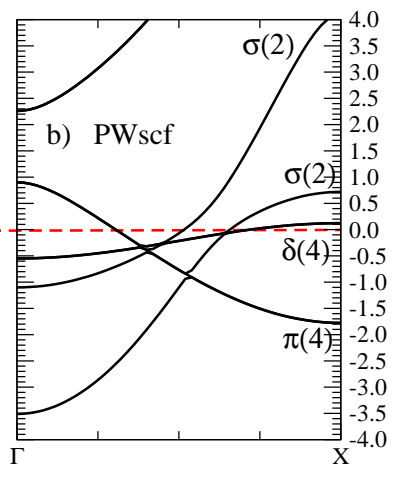

k

FIG. 9: a) TBLDA and b) PWscf band structure of a non-magnetic Fe monatomic wire with an interatomic distance of 4.29 a.u.. Each band is labelled by its symmetry character and degeneracy (including spin).

The $\delta$ band is easily identified as being the flattest band which disperses positively. Indeed its dispersion relation is that of a linear chain of $d_{x y}$ (or $d_{x^{2}-y^{2}}$ ) orbitals with a small and negative hopping integral $d d \delta$. In Fe the $p$ level is much higher in energy than the $d$ level. As a consequence, the mixing between $p$ and $d$ orbitals is small and the $\pi$ bands split into a lower band with an almost pure $d_{z x}$ (or $d_{y z}$ ) character and a higher one with an almost pure $p_{x}$ or $p_{y}$ character. The first one shows a dispersion very similar to that of a linear chain of $d_{z x}$ (or $d_{y z}$ ) orbitals, i.e., it disperses negatively since the corresponding integral $d d \pi$ is positive. The second one disperses positively since $p p \pi$ is negative, this band is present in the PWscf calculation, but is outside the energy range of Fig.9 in the TBLDA results. This means that the TB parameters relative to $p$ bands are not very accurate but since the higher $\pi$ band is unoccupied, this inaccuracy will have no influence on our results for the ground state. The two remaining bands are the two lowest $\sigma$ bands. An analysis of the character of these bands using the TBLDA model shows that the lowest band has almost no $p$ character, the weight of $s$ and $d_{3 z^{2}-r^{2}}$ orbitals are almost the same at the $\Gamma$ point while at the $X$ point, the state is almost a pure $d$ one. For the next $\sigma$ band the $d_{3 z^{2}-r^{2}}$ character decreases continuously from $\simeq 0.5$ to 0 along $\Gamma X$, the $p_{z}$ character increases continuously from 0 to 1 while the $s$ character is $\simeq 0.5$ at $\Gamma$, has a maximum at the midpoint and vanishes at the $X$ point.

\section{B. Magnetic transition}

As in the bulk we have studied the appearance of a magnetic moment when the interatomic distance $d$ increases using the TBLDA model as well as the PWscf code with GGA. As seen in Fig 10 a low spin state (LS) is found at short interatomic distances with a slowly increasing moment, then, around $d=3.8$ a.u., the moment increases abruptly to reach a high spin state (HS) corresponding to the saturated configuration. The agreement between both methods is quite satisfactory, in particular, the HS state appears at the same interatomic distance. It should however be 
mentioned that the TBGGA fails to reproduce the wire band structure in the range of interatomic distances involved at the transition due to an incorrect position of the $s$ level. Actually, for interatomic distances around 3.8 a.u. this $s$ level is pushed at higher energies and instead of a LS/HS transition one finds a NM/HS transition. Let us recall that the TB parameters are fitted on bulk results only, and for interatomic distances and coordinence larger than in the wire. Therefore, the extrapolation of the law giving the atomic levels as a function of the atomic environment may fail for the wire. From the above study it appears that the TBLDA levels are much better than the GGA ones. As a consequence, in the following, LDA parameters will be used.

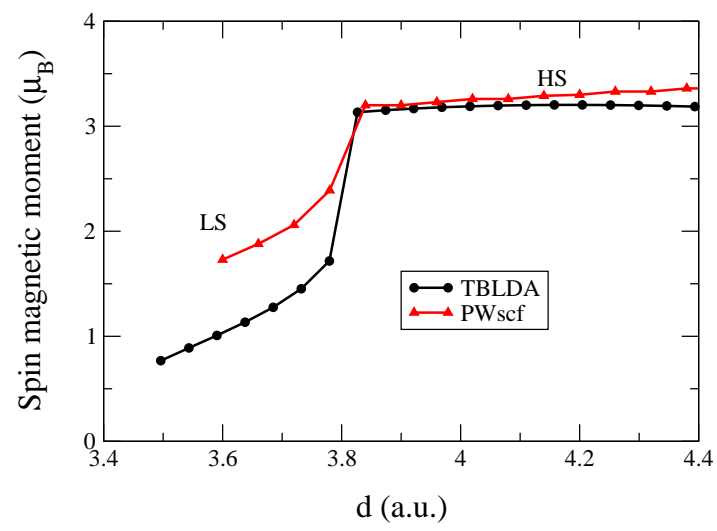

FIG. 10: TBLDA and PWscf spin moment of a monoatomic wire, as a function of the interatomic distance.

\section{Spin-orbit coupling effects}

1. Effects of spin-orbit coupling on the band structure from perturbation theory.

The removal of degeneracies due to the spin-orbit coupling can be predicted by perturbation theory. As seen in Fig.9 two types of degeneracies occur for the wire: in addition to spin degeneracy, either a band is degenerate for any value of $\mathbf{k}$ due to symmetry or the degeneracy is limited to band crossings. In the former case $H_{\text {so }}$ may produce a splitting of the bands while in the latter it may open a gap between the two crossing bands.

Let us first consider the non magnetic case for which the band structure should be independent of $\theta$ and $\varphi$. The $\delta$ band is four-fold degenerate. For $\theta=0(\pi / 2) H_{\text {so }}$ couples the $d_{x y}$ and $d_{x^{2}-y^{2}}$ orbitals with the same (opposite) spins with a coupling matrix element $\pm i \xi$ (see Appendix A). Consequently the $\delta$ band is unfolded symmetrically with a band splitting given by $2 \xi$. The same type of arguments applies to the lowest four-fold $\pi$ band if the small $p$ character is neglected but the coupling matrix element is now $\pm i \xi / 2$ and therefore the band splitting is equal to $\xi$. No removal of degeneracy is expected on the two-fold $\sigma$ bands since there is no matrix elements of $H_{\text {so }}$ between the $d_{3 z^{2}-r^{2}}$ orbitals of opposite spins. Finally in the unperturbed band structure (Fig.9) there are two crossing points with the $\sigma$ and $\delta$ bands which are not coupled by $H_{\text {so }}$. Thus there is no removal of degeneracy. On the contrary the other crossing points (between $\sigma$ and $\pi$ bands, or $\pi$ and $\delta$ bands) are avoided.

In the magnetic case the band structure is independent of $\varphi$ for symmetry reasons but depends on the polar angle $\theta$ when spin-orbit coupling is taken into account. This is the consequence of the removal of spin degeneracy in the 
unperturbed state. Indeed for $\theta=0, H_{\text {so }}$ couples states with the same spin and a splitting $2 \xi(\xi)$ exists in the $\delta$ (lowest $\pi$ ) bands, for both spins, while for $\theta=\pi / 2$, the bands of up and down spins being well separated in energy, $H_{\text {so }}$ has a negligible effect on the $\delta$ and lowest $\pi$ bands. Finally, removals of degeneracy are expected at some crossing points. The number of opened gaps should be small at $\theta=0$ since, for bands of different symmetries, only states with opposite spins may be coupled by $H_{\text {so }}$. As the exchange splitting is large the number of avoided crossings should be very small. On the contrary at $\theta=\pi / 2$ a detailed analysis of the $H_{\text {so }}$ matrix (see Appendix A) reveals that states with the same as well as different spins may be coupled and the number of avoided crossings is expected to be larger than for $\theta=0$.

The TBLDA calculation is presented in Fig. 11 for a magnetization parallel $(\theta=0)$ or perpendicular $(\theta=\pi / 2)$ to the wire. The results are in perfect agreement with the predictions of perturbation theory. Note that a rather good overall agreement is also obtained with PWscf calculations 45. In particular the splitting of the $\delta$ band in the latter calculations is $120 \mathrm{meV}$, i.e., exactly $2 \xi$ which confirms that spin-orbit coupling is an intra-atomic effect and that $\xi$ is a purely atomic quantity, i.e., it does not depend on the atomic environment.

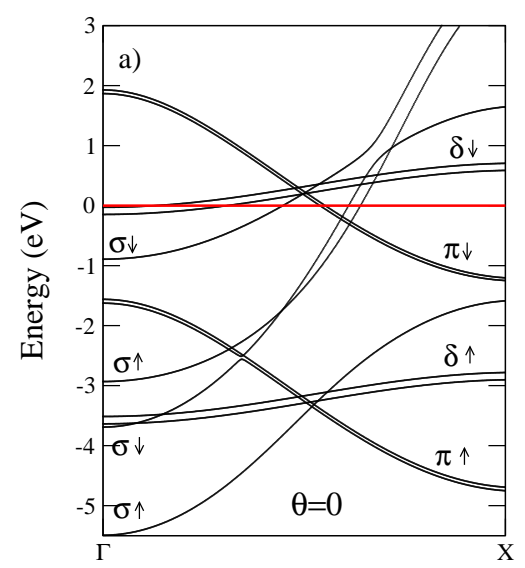

$\mathrm{k}$

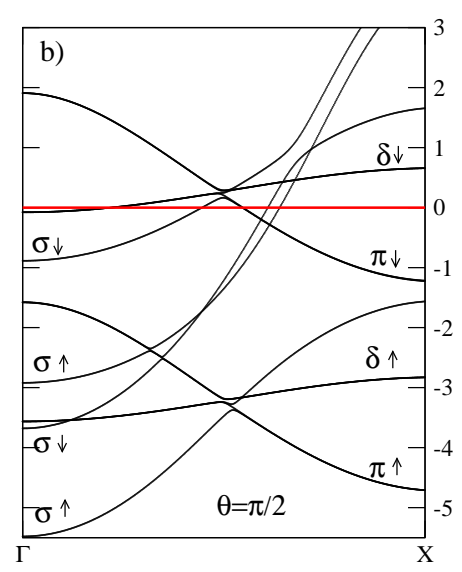

$\mathrm{k}$

FIG. 11: TBLDA band structure including spin-orbit coupling for a magnetic Fe monatomic wire (interatomic distance d=4.16 a.u.) with a magnetization a) parallel and b) perpendicular to the wire.

\section{Magnetocrystalline anisotropy.}

Since the monatomic wire is a one dimensional system with the lowest possible coordination we expect a magnetocrystalline anisotropy larger than at the surface. Moreover, due to the axial symmetry of the wire the energy will only depend on the angle $\theta$ between the magnetization and the axis of the wire. By imposing the symmetry properties of the wire to the quadratic form in $l, m$, and $n$ giving the magnetocrystalline anisotropy in second order perturbation theory, we find:

$$
\Delta E_{i}^{(2)}(\theta, \varphi)-\Delta E_{i}^{(2)}(0,0)=K_{1}^{\prime} \sin ^{2} \theta
$$

In Fig. 12 we present the results of our TBLDA and PWscf calculations obtained at various interatomic distances. 
The variation of energy with $\theta$ is perfectly fitted by the above equation. Interestingly an inversion of the easy axis is observed (i.e., a change of sign of $K_{1}^{\prime}$ ). Indeed, for interatomic distances $d$ larger than 3.78 a.u. (3.93 a.u. with PWscf) the easy axis is along the wire, while it is perpendicular when $d$ is smaller. This inversion of the easy axis occurs at the LS/HS magnetic transition. A detailed analysis of the charge distribution shows that the magnetic transition is accompanied by a noticeable change of filling of the $\delta$ bands.

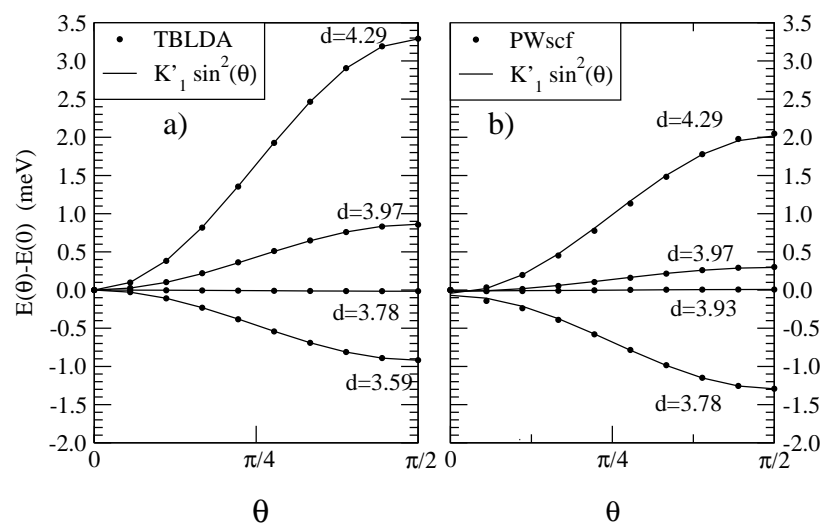

FIG. 12: Variation of the magnetocrystalline anisotropy (per atom) of a monatomic wire as a function of the angle $\theta$ from a TBLDA calculation (left panel) and PWscf calculation (right panel) for various interatomic distances $d$ (in a.u.).

\section{Orbital moment.}

Let us first derive the expressions of the components of the orbital moment relative to the spin framework using perturbation theory. In a monatomic wire of atoms with only $d$ orbitals, the band hamiltonian is reduced to five decoupled linear chains with a single orbital on each atom, thus $\mathcal{N}_{\lambda \lambda^{\prime} \sigma}(\mathbf{k}, E)=\mathcal{N}_{\lambda \lambda \sigma}(\mathbf{k}, E) \delta_{\lambda \lambda^{\prime}}$ and Eq. 36 becomes:

$$
<\mathbf{L}_{i}^{\prime \prime}>=-2 \xi \sum_{\lambda \mu} \sum_{\sigma}\left\langle\bar{\lambda} \sigma\left|\mathbf{L}_{i}^{\prime \prime}\right| \bar{\mu} \sigma\right\rangle\langle\bar{\lambda} \sigma|\mathbf{L} \cdot \mathbf{S}| \bar{\mu} \sigma\rangle^{*} I_{\lambda \mu}
$$

where $I_{\lambda \mu}$ denotes the term in Eq.36 involving the summation over $\mathbf{k}$ but with $\lambda^{\prime}=\lambda$ and $\mu^{\prime}=\mu$.

In order to find the angular dependence of $\left\langle\mathbf{L}_{i}^{\prime \prime}\right\rangle$, we use Eq.18 with the matrix elements of $\mathbf{L}_{i}$ and of $H_{s o}$ given in Appendix A taking $\varphi=0$, for simplicity, since the wire has an axial symmetry. Then it is easily seen that:

$$
\begin{aligned}
& <L_{i x^{\prime \prime}}>=K_{1 x^{\prime \prime}} \sin 2 \theta \\
& <L_{i z^{\prime \prime}}>=K_{0 z^{\prime \prime}}+K_{1 z^{\prime \prime}} \sin ^{2} \theta
\end{aligned}
$$

while $\left\langle L_{i y^{\prime \prime}}>=0\right.$ as expected since $y^{\prime \prime}$ is perpendicular to the plane defined by the wire and the spin quantization axis.

The results of the TBLDA calculations of $\left\langle L_{i x^{\prime \prime}}\right\rangle$ and $\left\langle L_{i z^{\prime \prime}}\right\rangle$, shown in Fig. 13 for an interatomic distance $d=4.29$ a.u., are in perfect agreement with this analysis. Note that $\left\langle L_{i z^{\prime \prime}}\right\rangle$ can reach a value as large as 
$0.42 \mu_{B}$ when the magnetization is along the wire and that the anisotropy of the orbital moment $<L_{i z^{\prime \prime}}(\theta=\pi / 2)>$ $-<L_{i z^{\prime \prime}}(\theta=0)>=-0.22 \mu_{B}$, is rather large. At the same interatomic distance (see Fig. 12) the corresponding magnetocrystalline anisotropy is $3.3 . \mathrm{meV}$. Thus the ratio of this anisotropy to that of the orbital moment is equal to $-15 \mathrm{meV}$ which is in perfect agreement with Eq.38 $(-\xi / 4=-15 \mathrm{meV})$. This was rather expected since the spin up $d$ bands are completely filled and well separated $(\simeq 3 e V)$ from the spin down ones at this interatomic distance, both bands being much narrower than at the surface.

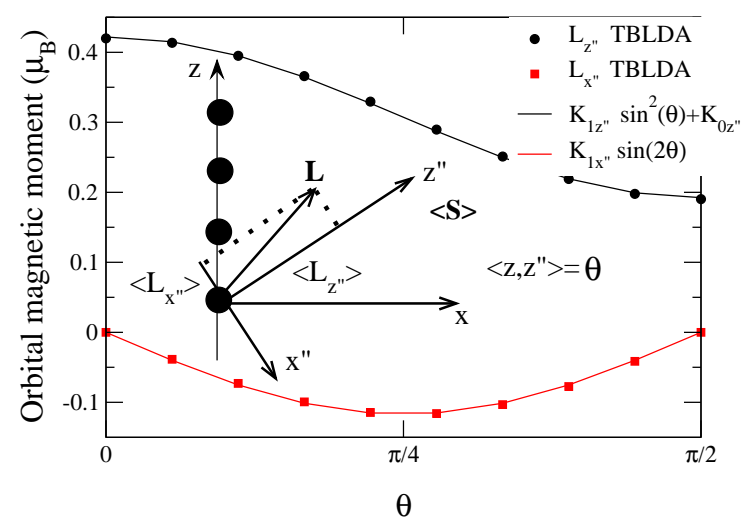

FIG. 13: Variation of the components of the orbital moment relative to the spin framework for a monatomic wire (interatomic distance $d=4.29$ a.u.) as a function of the direction of magnetization given by the angle $\theta$ obtained with TBLDA parameters.

At shorter distances for which the LS state is found, Eq.38 is no longer valid and even does not predict the sign of this ratio since the magnetocrystalline anisotropy changes sign, contrary to that of the orbital moment.

Finally let us note that in the presence of spin-orbit coupling the spin moment is very slightly anisotropic: typically $<S_{z "}$ " $>$ varies by about $0.02 \mu_{B}$ between $\theta=0$ and $\theta=\pi / 2$. Moreover, similarly to the orbital moment, a small component $<S_{x "}>$ is present when $\left.\theta \in\right] 0, \pi / 2[$.

\section{CONCLUSION}

We have shown that starting from the parametrized spd TB model set up by Mehl and Papaconstantopoulos [21], adding a Stoner-like spin polarization term and a spin-orbit coupling term, i.e, introducing only two additional parameters $I_{d d^{\prime}}$ and $\xi$, we have been able to describe in detail the magnetic properties (spin moments, orbital moments and magnetocrystalline anisotropy energy) of iron in systems of various dimensionalities and coordinences. Whenever possible the results have been compared with those of the PWscf code or other existing theoretical or experimental data, and the agreement is excellent. Our simple TB model allowed us to derive some new results. For example in the case of surfaces we have studied the variation of the component of the orbital magnetic moment on the spin quantization axis $\left\langle L_{z}\right.$ " $>$ as a function of depth, and shown that the enhancement of this quantity is still noticeable on the second layer for the (001) surface but almost cancels on the third and innermost layers. In the wire we have found that the easy axis of magnetization is along the wire at the theoretical equilibrium distance but can switch to the perpendicular direction under compression. Moreover $<L_{z}$ " $>$ is strongly enhanced and highly anisotropic since for $z "$ along the wire it is about $0.42 \mu_{B}$ and decreases to half this value for the perpendicular direction. In addition 
for intermediate orientations the orbital moment has a non-negligible component $<L_{x}$ " $>$ perpendicular to $z$ " and in the plane made by the wire and $z "$. This component is also anisotropic and proportional to $\sin 2 \theta$ as predicted by perturbation theory. Finally we have shown that the law of Bruno 8 relating the MAE and the anisotropy of the orbital moment, is perfectly obeyed for the wire at equilibrium distance, which is not the case for the surfaces, even for the (001) orientation at which the magnetic moment is saturated.

The success of our model opens up the possibility of obtaining accurate results on other elements and systems with much more complex geometries.

\section{Acknowledgments}

It is our pleasure to thank M. Viret for very fruitful discussions and A. Dal Corso and A. Smogunov for precious advice concerning the PWscf code.

\section{APPENDIX A: THE MATRIX ELEMENTS OF THE ORBITAL MOMENT AND SPIN-ORBIT COUPLING OPERATORS}

The matrix elements $\left\langle\bar{\lambda} \sigma|\mathbf{L} . \mathbf{S}| \bar{\mu} \sigma^{\prime}\right\rangle$, where $\bar{\lambda}$ and $\bar{\mu}$ are the angular parts of $d$ orbitals $\left(d_{x y}, d_{y z}, d_{z x}, d_{x^{2}-y^{2}}, d_{3 z^{2}-r^{2}}\right)$ centered at the same site as the angular momentum operator, can be easily calculated as a function of the direction (defined by the polar and azimuthal angles $\theta$ and $\varphi$ relative to the crystal axes) of the spin quantization axis $z^{\prime \prime}$ which is often taken in the magnetization direction that may be different from the $z$ axis of the crystal. This is achieved by choosing a new coordinate framework $x^{\prime \prime}, y^{\prime \prime}, z^{\prime \prime}$ referred to as the spin framework which is obtained by rotating first the $x y$ axes of the crystal by the angle $\varphi$ around $z$. This gives a new framework $x^{\prime}, y^{\prime}, z^{\prime}$ which is then rotated by the angle $\theta$ around $y^{\prime}$. Using the matrix elements of $\mathbf{L}$ in the basis of real $\mathrm{d}$ orbitals:

$$
\begin{aligned}
\left\langle\lambda \sigma\left|L_{x}\right| \mu \sigma\right\rangle= & \left(\begin{array}{ccccc}
0 & 0 & -i & 0 & 0 \\
0 & 0 & 0 & -i & -i \sqrt{3} \\
i & 0 & 0 & 0 & 0 \\
0 & i & 0 & 0 & 0 \\
0 & i \sqrt{3} & 0 & 0 & 0
\end{array}\right) \\
\left\langle\lambda \sigma\left|L_{y}\right| \mu \sigma\right\rangle & =\left(\begin{array}{ccccc}
0 & i & 0 & 0 & 0 \\
-i & 0 & 0 & 0 & 0 \\
0 & 0 & 0 & -i & i \sqrt{3} \\
0 & 0 & i & 0 & 0 \\
0 & 0 & -i \sqrt{3} & 0 & 0
\end{array}\right)
\end{aligned}
$$




$$
\left\langle\lambda \sigma\left|L_{z}\right| \mu \sigma\right\rangle=\left(\begin{array}{ccccc}
0 & 0 & 0 & 2 i & 0 \\
0 & 0 & i & 0 & 0 \\
0 & -i & 0 & 0 & 0 \\
-2 i & 0 & 0 & 0 & 0 \\
0 & 0 & 0 & 0 & 0
\end{array}\right)
$$

one finds:

$$
\begin{aligned}
& \langle\bar{\lambda} \uparrow|\mathbf{L} . \mathbf{S}| \bar{\mu} \uparrow\rangle=\left(\begin{array}{ccccc}
0 & \frac{i}{2} \sin \varphi \sin \theta & \frac{-i}{2} \cos \varphi \sin \theta & i \cos \theta & 0 \\
\frac{-i}{2} \sin \varphi \sin \theta & 0 & \frac{i}{2} \cos \theta & \frac{-i}{2} \cos \varphi \sin \theta & \frac{-i \sqrt{3}}{2} \cos \varphi \sin \theta \\
\frac{i}{2} \cos \varphi \sin \theta & \frac{-i}{2} \cos \theta & 0 & \frac{-i}{2} \sin \varphi \sin \theta & \frac{i \sqrt{3}}{2} \sin \varphi \sin \theta \\
-i \cos \theta & \frac{i}{2} \cos \varphi \sin \theta & \frac{i}{2} \sin \varphi \sin \theta & 0 & 0 \\
0 & \frac{i \sqrt{3}}{2} \cos \varphi \sin \theta & \frac{-i \sqrt{3}}{2} \sin \varphi \sin \theta & 0 & 0
\end{array}\right) \\
& \langle\bar{\lambda} \uparrow|\mathbf{L} . \mathbf{S}| \bar{\mu} \downarrow\rangle=\left(\begin{array}{ccccc}
0 & \frac{1}{2} f(\varphi, \theta) & \frac{1}{2} g(\varphi, \theta) & -i \sin \theta & 0 \\
-\frac{1}{2} f(\varphi, \theta) & 0 & \frac{-i}{2} \sin \theta & \frac{1}{2} g(\varphi, \theta) & \frac{\sqrt{3}}{2} g(\varphi, \theta) \\
-\frac{1}{2} g(\varphi, \theta) & \frac{i}{2} \sin \theta & 0 & -\frac{1}{2} f(\varphi, \theta) & \frac{\sqrt{3}}{2} f(\varphi, \theta) \\
i \sin \theta & -\frac{1}{2} g(\varphi, \theta) & \frac{1}{2} f(\varphi, \theta) & 0 & 0 \\
0 & -\frac{\sqrt{3}}{2} g(\varphi, \theta) & -\frac{\sqrt{3}}{2} f(\varphi, \theta) & 0 & 0
\end{array}\right)
\end{aligned}
$$

where $f(\varphi, \theta)=\cos \varphi+i \sin \varphi \cos \theta$ and $g(\varphi, \theta)=\sin \varphi-i \cos \varphi \cos \theta$. The other blocks of the $(10 \times 10)$ spin-orbit matrix are obtained from the relations:

$$
\begin{aligned}
\langle\bar{\lambda} \downarrow|\mathbf{L} \cdot \mathbf{S}| \bar{\mu} \uparrow\rangle & =-\langle\bar{\lambda} \uparrow|\mathbf{L} \cdot \mathbf{S}| \bar{\mu} \downarrow\rangle^{*} \\
\langle\bar{\lambda} \downarrow|\mathbf{L} \cdot \mathbf{S}| \bar{\mu} \downarrow\rangle & =\langle\bar{\lambda} \uparrow|\mathbf{L} \cdot \mathbf{S}| \bar{\mu} \uparrow\rangle^{*}
\end{aligned}
$$

in which $*$ denotes the complex conjugate. In addition a very useful relation has been derived by Bruno[6]:

$$
\operatorname{Re}\left[\langle\bar{\lambda} \uparrow|\mathbf{L} . \mathbf{S}| \bar{\mu} \downarrow\rangle\left\langle\bar{\mu}^{\prime} \downarrow|\mathbf{L} . \mathbf{S}| \bar{\lambda}^{\prime} \uparrow\right\rangle\right]+\langle\bar{\lambda} \uparrow|\mathbf{L} . \mathbf{S}| \bar{\mu} \uparrow\rangle\left\langle\bar{\mu}^{\prime} \uparrow|\mathbf{L} . \mathbf{S}| \bar{\lambda}^{\prime} \uparrow\right\rangle=\text { Cst. }
$$

Let us note however that the spin quantization axis could have been taken along the $z$ axis of the crystal, in which case the spin-orbit matrix elements would be given by the above matrices with $\theta=\varphi=0$ but the spin polarized term of the Hamiltonian becomes:

$$
-\frac{1}{4}\left[\left(\Delta_{\lambda}^{i}+\Delta_{\mu}^{j}\right) \mathcal{S}_{i j}^{\lambda \mu}\right] \otimes\left(\begin{array}{cc}
\cos \theta & \exp (-i \varphi) \sin \theta \\
\exp (i \varphi) \sin \theta & -\cos \theta
\end{array}\right)
$$

where $\otimes$ means the direct product of matrices. 
In the case of collinear spins and in the presence of spin-orbit coupling the first point of view is more convenient to treat spin-orbit coupling effects within perturbation theory since $H_{\text {so }}$ describes the perturbation completely. The second point of view is preferable when dealing with non-collinear spins. Indeed this avoids the transformation of the inter-atomic part of $H_{T B H F}$ since, in that case, the spin functions are not the same at the two sites.

\section{APPENDIX B: CALCULATION OF THE ORBITAL MOMENT FOR A NON-ORTHOGONAL BASIS SET}

Let us generalize Eq.17 to take overlap into account. If we note that the integral in Eq.13 gives the population of the spin-orbital $|i l m \sigma\rangle$, an obvious generalization is to replace this population by the Mulliken one. Thus $\rho_{i l m \sigma}(E)$ becomes:

$$
\rho_{i l m \sigma}(E)=\operatorname{Re} \sum_{\substack{i^{\prime} l^{\prime} m^{\prime} \\ n}} a_{i l m \sigma}^{n *} a_{i^{\prime} l^{\prime} m^{\prime} \sigma}^{n} \mathcal{S}_{i i^{\prime}}^{l m}, l^{\prime} m^{\prime} \delta\left(E-E_{n}\right)
$$

with:

$$
\left|\psi_{n}\right\rangle=\sum_{i l m \sigma} a_{i l m \sigma}^{n} \mid i l m \sigma>
$$

and

$$
\mathcal{S}_{i i^{\prime}}^{l m, l^{\prime} m^{\prime}}=\left\langle i l m \sigma \mid i^{\prime} l^{\prime} m^{\prime} \sigma\right\rangle
$$

thus

$$
<L_{i z^{\prime \prime}}>=\operatorname{Re} \sum_{\substack{l m, i^{\prime} l^{\prime} m^{\prime} \sigma \\ n \text { occ }}} m a_{i l m \sigma}^{n *} a_{i^{\prime} l^{\prime} m^{\prime} \sigma}^{n} \mathcal{S}_{i i^{\prime}}^{l m} l^{\prime} m^{\prime}
$$

and, after simple algebraic manipulations:

$$
\left.<L_{i z^{\prime \prime}}\right\rangle=\operatorname{Re} \sum_{\substack{l m, i^{\prime} l^{\prime} m^{\prime} \sigma \\ n \text { occ }}}\left\langle\psi_{n} \mid i l m \sigma\right\rangle m\left[\mathcal{S}^{-1}\right]_{i i^{\prime}}^{l m, l^{\prime} m^{\prime}}\left\langle i^{\prime} l^{\prime} m^{\prime} \sigma \mid \psi_{n}\right\rangle
$$

The generalization of Eq. 16 yields:

$$
<\mathbf{L}_{i}^{\prime \prime}>=\operatorname{Re} \sum_{l m, l^{\prime \prime}}\left\langle\psi_{n} \mid i l m \sigma\right\rangle\left[\mathbf{L}_{i}^{\prime \prime}\right]_{l m, l^{\prime \prime} m^{\prime \prime}, i^{\prime} l^{\prime} m^{\prime} \sigma}\left[\mathcal{S}^{-1}\right]_{i i^{\prime}}^{l^{\prime \prime} m^{\prime \prime}, l^{\prime} m^{\prime}}\left\langle i^{\prime} l^{\prime} m^{\prime} \sigma \mid \psi_{n}\right\rangle
$$

and, in the basis $|i \lambda \sigma\rangle$ :

$$
<\mathbf{L}_{i}^{\prime \prime}>=\operatorname{Re} \sum_{\substack{\lambda, \mu, i^{\prime} \nu \sigma \\ n \text { occ }}}\left\langle\psi_{n} \mid i \lambda \sigma\right\rangle\left[\mathbf{L}_{i}^{\prime \prime}\right]_{\lambda \mu}\left[\mathcal{S}^{-1}\right]_{i i^{\prime}}^{\mu \nu}\left\langle i^{\prime} \nu \sigma \mid \psi_{n}\right\rangle
$$


with:

$$
\left\langle i^{\prime} \nu \sigma \mid \psi_{n}\right\rangle=\sum_{i \lambda \sigma} c_{i \lambda \sigma}^{n} \mathcal{S}_{i^{\prime} i}^{\nu \lambda}
$$

so that we get finally:

$$
<\mathbf{L}_{i}^{\prime \prime}>=\operatorname{Re} \sum_{\substack{\lambda \mu i^{\prime} \nu \sigma \\ n \text { occ }}} c_{i \lambda \sigma}^{n *}\left[\mathbf{L}_{i}^{\prime \prime}\right]_{\lambda \mu} \mathcal{S}_{i i^{\prime}}^{\mu \nu} c_{i^{\prime} \nu \sigma}^{n}
$$

[1] Barreteau C, Guirado-López R, Spanjaard D, Desjonquères M-C and Oleś A M 2000 Phys. Rev. B 617781

[2] Barreteau C, Desjonquères M-C, Oleś A M and Spanjaard D 2004 Phys. Rev. B 69064432

[3] Friedel J, Lenglart P and Leman G 1964 J. Phys. Chem. Solids 25781

[4] Stearns M B, in 3d, 4d, and 5d Elements, Alloys and Compounds, edited by H. P. J. Wijn, Landolt-Börnstein, New Series, Group III, Vol. 19, Pt. a (Springer, Berlin, 1986) p. 34.

[5] Gradmann U, in Ferromagnetic Materials, edited by K.H.J. Buschow (Elsevier, Amsterdam, 1993), Vol. 7.

[6] Bruno P, PhD Thesis Orsay (1989), available at http://tel.ccsd.cnrs.fr/, and references therein

[7] Magnetism and Synchrotron Radiation Series: Lecture Notes in Physics, Vol. 565 Beaurepaire E, Scheurer F, Krill G, Kappler J-P (Eds.) 2001, XIV, 396 p., Hardcover

[8] Bruno P 1989 Phys. Rev. B 39865

[9] Gay J G and Richter R 1986 Phys. Rev. Lett. 56, 2728

[10] Wang D-S, Wu R and Freeman A J 1993 Phys. Rev. B 4714932

[11] Cinal M, Edwards D M and Mathon J 1994 Phys. Rev. B 50, 3754

[12] Lessard A, Moos T H and Hübner W 1997 Phys. Rev. B 562594

[13] Burkert T, Eriksson O, Simak S I, Ruban A V, Sanyal B, Nordström L and Wills J M 2005 Phys. Rev. B 71134411

[14] Qian X and Hübner W 1997 Phys. Rev. B 64092402

[15] K. Kyuno, J.-G. Ha, R. Yamamoto, and S. Asano, Phys. Rev. B 54, 1092 (1996).

[16] Broddefalk A, Nordblad P, Blomquist P, Isberg P, Wäppling R, Le Bacq 0 and Eriksson 0 2002 J. Magn. Magn. Mater. 241260

[17] Guirado-López R A, Dorantes-Dávila J and Pastor G M 2003 Phys. Rev. Lett. 90226402

[18] Dorantes-Dávila J, Dreyssé H and Pastor G M 1997 Phys. Rev. B 5515033

[19] Popescu V, Ebert H, Nonas B and Dederichs P H 2001 Phys. Rev. B 64184407

[20] Eriksson O, Nordström L, Pohl A, Severin L, Boring A M and Johansson B 1990 Phys. Rev. B 4111807

[21] Mehl M J and Papaconstantopoulos D A 1996 Phys. Rev. B 544519

[22] Slater J C and Koster G F 1954 Phys. Rev. 941498

[23] http://cst-www.nrl.navy.mil/bind/.

[24] van de Laan G 1998 J. Phys.: Condens. Matter 103239

[25] Baroni S, Dal Corso A, de Gironcoli S, Giannozzi P, Cavazzoni C, Ballabio G, Scandolo S, Chiarotti G, Focher P, Pasquarello A, Laasonen K, Trave A, Car R, Marzari N, Kokalj A http://www.pwscf.org/.

[26] Macedo W A A and Keune W 1998 Phys. Rev. Lett. 61475

[27] Torija M A, Gai Z, Myoung N, Plummer E W and Shen J 2005 Phys. Rev. Lett. 95027201 
[28] Kübler J 1981 Physics Letters 81 A 81

[29] Moruzzi V L 1986 Phys. Rev. Lett. 57221

[30] Herper H C, Hoffmann E and Entel P 1999 Phys. Rev. B 603839

[31] Knöpfle K, Sandratskii L M and Kübler J 2000 Phys. Rev. B 625564

[32] Steinle-Neumann G, Cohen R E and Stixrude L 2004 J. Phys.: Cond. Matt. 16 S1109

[33] Moruzzi V L, Marcus P M and Kübler J 1989 Phys. Rev. B 396957

[34] Wang C S, Klein B M and Krakauer K 1985 Phys. Rev. Lett. 541852

[35] Stearns M B in Landolt-Börnstein Numerical Data and Functional Relationships in Science and Technology, edited by Wijn H P J(Springer-Verlag, Berlin, 1986), Group 3, Vol. 19, Pt. a; Bonnenberg D, Hempel K A and Wijn H P J, ibid.

[36] Barreteau C, Spanjaard D and M-C Desjonquères 1998 Phys. Rev. B 589721

[37] Kim H-J, Vescovo E, Heinze S and Blügel S 2001 Surf. Sci. 478193

[38] Eriksson 0, Fernando G W, Albers R C and Boring A M Solid State 1991 Communications 78801

[39] Tischer M, Hjorstam O, Arvanitis D, Hunter Dunn J, May F, Baberschke K, Trygg J, Wills J M, Johansson B and Eriksson O 1995 Phys. Rev. Lett. 751602

[40] Mokroussov Y, Bihlmayer G and Blügel S 2005 Phys. Rev. B 72045402

[41] Delin A, Tosatti E and Weht R 2004 Phys. Rev. Lett. 92057201

[42] Delin A and Tosatti E 2004 Surf. Sci. 566-568 262

[43] Delin A and Tosatti E 2004 J. Phys.: Cond. Matt. 168061

[44] Smit R H M, Untiedt C, Yanson A I and van Ruitenbeek J M 2001 Phys. Rev. Lett. 87266102 and references therein.

[45] Viret M, Gabureac M, Ott F, Fermon C, Barreteau C and Guirado-Lopez R A to be published, cond-mat/0602298. 